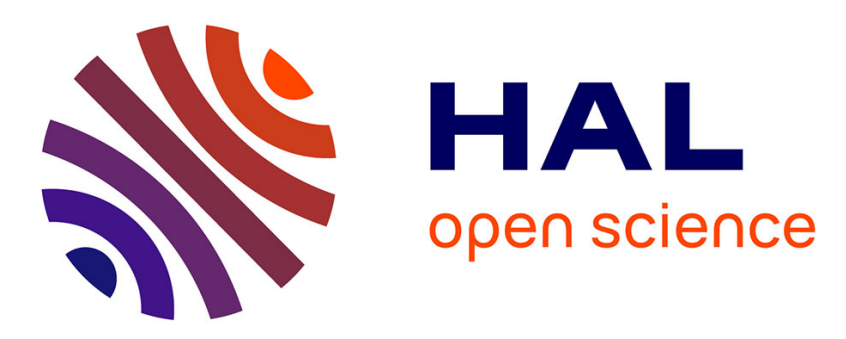

\title{
Analysis of the PSPG method for the transient Stokes' problem
}

Erik Burman, Miguel Angel Fernández

\section{To cite this version:}

Erik Burman, Miguel Angel Fernández. Analysis of the PSPG method for the transient Stokes' problem. Computer Methods in Applied Mechanics and Engineering, 2011, 200 (41-44), pp.28822890. 10.1016/j.cma.2011.05.001 . inria-00426777v3

\section{HAL Id: inria-00426777 https://hal.inria.fr/inria-00426777v3}

Submitted on 12 Apr 2011

HAL is a multi-disciplinary open access archive for the deposit and dissemination of scientific research documents, whether they are published or not. The documents may come from teaching and research institutions in France or abroad, or from public or private research centers.
L'archive ouverte pluridisciplinaire HAL, est destinée au dépôt et à la diffusion de documents scientifiques de niveau recherche, publiés ou non, émanant des établissements d'enseignement et de recherche français ou étrangers, des laboratoires publics ou privés. 


\section{N R I A}

INSTITUT NATIONAL DE RECHERCHE EN INFORMATIQUE ET EN AUTOMATIQUE

\section{Analysis of the PSPG method for the transient Stokes'problem}

Erik Burman — Miguel A. Fernández

$\mathbf{N}^{\circ} \mathbf{7 0 7 4}$

October 2009

Thème $\mathrm{BIO}$

apport

de recherche 



\title{
RIN RIA
}

\section{Analysis of the PSPG method for the transient Stokes' problem}

\author{
Erik Burman, Miguel A. Fernándeđ† \\ Thème BIO - Systèmes biologiques \\ Projet REO \\ Rapport de recherche $\mathrm{n}^{\circ} 7074$ - October $2009-21$ pages
}

\begin{abstract}
We propose a new analysis for the PSPG method applied to the transient Stokes' problem. Stability and convergence are obtained under different conditions on the discretization parameters depending on the approximation used in space. For the pressure we prove optimal stability and convergence only in the case of piecewise affine approximation under the standard condition on the time-step. Finally, we show that the stability problems of the PSPG method can be circumvented using an appropriate discrete reconstruction of the Laplacian operator.
\end{abstract}

Key-words: Transient Stokes' equations, finite element methods, PSPG stabilization, time discretization, Ritz-projection.

* University of Sussex, UK; e-mail: E.N.Burman@sussex.ac.uk

${ }^{\dagger}$ INRIA, REO team; e-mail: miguel.fernandez@inria.fr 


\section{Analyse de la méthode PSPG pour l'équation de Stokes transitoire}

Résumé : Nous proposons une nouvelle analyse pour la méthode PSPG appliquée à l'équation de Stokes transitoire. On montre la stabilité et la convergence de la méthode sous différentes conditions et selon le type d'approximation en space. Pour la pression, stabilité et convergence optimale sont établies dans le cas d'approximations affines par morceaux, sous une condition standard de type CFL parabolique inverse. Finalement, on montre que les problèmes de stabilité de la méthode PSPG peuvent être contournés en utilisant une reconstruction discrète appropriée de l'opérateur Laplacien.

Mots-clés : Équation de Stokes transitoire, méthode d'éléments finis, stabilisation PSPG, discrétisation en temps, projection de Ritz. 


\section{Introduction}

The pressure stabilized Petrov-Galerkin (PSPG) method, as introduced by Hughes et al. in [16, is a popular tool for the approximation of the Stokes' problem using equal order interpolation for velocities and pressures. In spite of its extensive use, so far it has been analyzed for the transient case only for the velocities, using the backward Euler scheme and under an inverse CFL-condition. If $\tau$ and $h$ denote the discretization parameters, in time and space respectively, and $\nu$ denotes the kinematic viscosity, the condition writes $h^{2} \leq \nu \tau$. To our best knowledge, the first reference where this condition appeared was in the work by Picasso and Rappaz [18, (see also 2]). The small time-step instability was thoroughly investigated by Bochev et al. in [7, 6, where they examined the algebraic properties of the system matrices. Their conclusion was that regularity of the system imposed an inverse parabolic CFL condition. They also observed numerically, in [5], that for higher polynomial order the instability polluted the velocity approximation as well.

Our aim in this paper is to consider the transient Stokes' equations discretized using Petrov-Galerkin pressure stabilization and derive global in time stability, using the variational framework. From this different viewpoint we arrive at similar conclusions as [6], but with global stability bounds that we then use to derive optimal order error estimates. The estimates for the velocities also gives insight in a possible mechanism for the observed loss of accuracy in the velocity approximation for higher order polynomials reported in 5 .

The case of symmetric stabilization methods for the transient Stokes' problem was treated in [9. There we proved, that for symmetric stabilizations, the small time-step instability can be circumvented by using a particular discrete initial data given by the Ritz-projection associated to the discrete Stokes' operator. Our analysis herein shows that also for the PSPG method the small time-step pressure instability stems from the initial data, however it can not be cured using the Ritz-projection. The reason for this is a coupling between the time-derivative and the pressure gradient, appearing in the estimate for the acceleration, resulting in the factor $h^{2} /(\nu \tau)$ in the stability estimate.

For the global in time stability estimate for the velocities, we observe that the properties of the PSPG method change drastically depending on what approximation spaces are used. Indeed, depending on the regularity of the discretization space, different conditions must be imposed in order for stability to hold. For high-order polynomial spaces, it appears that energy contributions from gradient jumps over element faces may interact with the time-derivative of the velocity and destabilize the solution if the time-step is too small. Since the gradient jumps are small for smooth solutions this instability may be difficult to observe numerically. We give one example of a computation where the velocity approximation diverges as the time-step is reduced.

The main theoretical results for the velocities are as follows:

- The backward Euler method (BDF1): stability and optimal convergence hold unconditionally for piecewise affine approximation. For higher polynomial orders the results hold under the inverse CFL-condition $\tau \geq h^{2} / \nu$.

- Crank-Nicolson and the second order backward differentiation method (BDF2) are unconditionally stable and have optimally convergent velocity 
approximation for piecewise affine approximation. For higher polynomial order stability and convergence holds under the condition $\tau \geq h / \nu^{\frac{1}{2}}$.

- All $C^{1}(\Omega)$ approximation spaces, such as the one obtained using NURBS, result in unconditionally stable PSPG methods for all the time-discretization schemes proposed above.

Note that under the above conditions, for each case the pressure approximation exists, but stability is obtained only in a weak norm. In particular this means that for piecewise affine approximation or $C^{1}$ approximation, the system matrix that must be inverted for every timestep is regular independent of the discretization parameters. We give the stability analysis of the velocities for all the above mentioned methods. For the sake of conciseness, the convergence is only proved in the case of the first- and the second-order backward differentiation formulas. The extension to the Crank-Nicolson scheme is straightforward.

As regards the pressure, stability and optimal convergence (in the natural norm) are proved, under the standard condition $\tau \geq h^{2} / \nu$, for piecewise affine approximation spaces and the BDF1 scheme. The extension to the BDF2 method is straightforward using the same techniques. Nevertheless, the case of high-order polynomials or the Crank-Nicolson method remains open.

As a remedy to the stability problems of the standard PSPG method we study a method using a reconstructed Laplacian proposed by Bochev and Gunzburger in [4. In this reference they show the improved stability properties of the PSPG method using a reconstructed Laplacian. Properties that we herein show extend to the transient problem. We also show, by proving equivalence of this method and the quasi-static orthogonal subscale (OSS) method (see [11, 1]), that this method indeed does not suffer from any instability, neither in the velocities nor in the pressure, provided boundary conditions are included in the reconstruction in a Nitsche-type fashion and the initial data is chosen suitably. This was shown for the OSS method in [9].

Although incomplete and possibly not sharp for high polynomial order, we hope that these result will bring some new insights in the dynamics of the PSPGmethod applied to the transient Stokes' problem. In particular, it is interesting to notice that the choice of space discretization seems important for the stability of the discretization of the transient problem.

\section{Problem setting}

Let $\Omega$ be a convex domain in $\mathbb{R}^{d}(d=2$ or 3$)$ with a polyhedral boundary $\partial \Omega$. For $T>0$ we consider the problem of solving, for $\boldsymbol{u}: \Omega \times(0, T) \longrightarrow \mathbb{R}^{d}$ and $p: \Omega \times(0, T) \longrightarrow \mathbb{R}$, the following time-dependent Stokes problem:

$$
\left\{\begin{aligned}
\partial_{t} \boldsymbol{u}-\nu \boldsymbol{\Delta} \boldsymbol{u}+\boldsymbol{\nabla} p=\boldsymbol{f}, & \text { in } \Omega \times(0, T), \\
\boldsymbol{\nabla} \cdot \boldsymbol{u}=0, & \text { in } \Omega \times(0, T), \\
\boldsymbol{u}=\mathbf{0}, & \text { on } \partial \Omega \times(0, T), \\
\boldsymbol{u}(\cdot, 0)=\boldsymbol{u}_{0}, & \text { in } \Omega .
\end{aligned}\right.
$$

Here, $\boldsymbol{f}: \Omega \times(0, T) \longrightarrow \mathbb{R}^{d}$ stands for the source term, $\boldsymbol{u}_{0}: \Omega \longrightarrow \mathbb{R}^{d}$ for the initial velocity and $\nu>0$ for a given constant viscosity. In order to introduce 
a variational setting for (1) we consider the following standard velocity and pressure spaces

$$
V \stackrel{\text { def }}{=}\left[H_{0}^{1}(\Omega)\right]^{d}, \quad H \stackrel{\text { def }}{=}\left[L^{2}(\Omega)\right]^{d}, \quad Q \stackrel{\text { def }}{=} L_{0}^{2}(\Omega),
$$

normed with

$$
\|\boldsymbol{v}\|_{H} \stackrel{\text { def }}{=}(\boldsymbol{v}, \boldsymbol{v})^{\frac{1}{2}}, \quad\|\boldsymbol{v}\|_{V} \stackrel{\text { def }}{=}\left\|\nu^{\frac{1}{2}} \nabla \boldsymbol{v}\right\|_{H}, \quad\|q\|_{Q} \stackrel{\text { def }}{=}\left\|\nu^{-\frac{1}{2}} q\right\|_{H},
$$

where $(\cdot, \cdot)$ (resp. $\left.(\cdot, \cdot)_{\partial \Omega}\right)$ denotes the standard inner product in $L^{2}(\Omega)$ (resp. $\left.L^{2}(\partial \Omega)\right)$. The standard seminorm of $H^{k}(\Omega)$ will be denoted by $|\cdot|_{k}$.

The transient Stokes' problem may be formulated in weak form as follows: For all $t>0$, find $\boldsymbol{u}(t) \in V$ and $p(t) \in Q$ such that

$$
\left\{\begin{aligned}
\left(\partial_{t} \boldsymbol{u}, \boldsymbol{v}\right)+a(\boldsymbol{u}, \boldsymbol{v})+b(p, \boldsymbol{v})=(\boldsymbol{f}, \boldsymbol{v}), & \text { a.e. in } \quad(0, T), \\
b(q, \boldsymbol{u})=0, & \text { a.e. in }(0, T), \\
\boldsymbol{u}(\cdot, 0)=\boldsymbol{u}_{0}, & \text { a.e. in } \Omega,
\end{aligned}\right.
$$

for all $\boldsymbol{v} \in V, q \in Q$ and with $a(\boldsymbol{u}, \boldsymbol{v}) \stackrel{\text { def }}{=}(\nu \boldsymbol{\nabla} \boldsymbol{u}, \boldsymbol{\nabla} \boldsymbol{v}), \quad b(p, \boldsymbol{v}) \stackrel{\text { def }}{=}-(p, \boldsymbol{\nabla} \cdot \boldsymbol{v})$.

From these definitions, the following classical coercivity and continuity estimates hold:

$$
a(\boldsymbol{v}, \boldsymbol{v}) \geq\|\boldsymbol{v}\|_{V}^{2}, \quad a(\boldsymbol{u}, \boldsymbol{v}) \leq\|\boldsymbol{u}\|_{V}\|\boldsymbol{v}\|_{V}, \quad b(\boldsymbol{v}, q) \leq\|\boldsymbol{v}\|_{V}\|q\|_{Q},
$$

for all $\boldsymbol{u}, \boldsymbol{v} \in V$ and $q \in Q$. It is known that if $\boldsymbol{f} \in C^{0}([0, T] ; H)$ and that $\boldsymbol{u}_{0} \in V \cap H_{0}(\operatorname{div} ; \Omega)$ problem (2) admits a unique solution $(\boldsymbol{u}, p)$ in $L^{2}(0, T ; V) \times$ $L^{2}(0, T ; Q)$ with $\partial_{t} \boldsymbol{u} \in L^{2}\left(0, T ; V^{\prime}\right)$ (see, e.g., [13]). Throughout this paper, $C$ stands for a generic positive constant independent of the discretization parameters and of $\nu$, but not of the mesh-geometry. We also use the notation $a \lesssim b$ meaning $a \leq C b$.

\section{Space and time discretization}

In this section we fully discretize problem (2), using the pressure stabilized Petrov-Galerkin method in space, and a (first- or second-order) backward difference formula or the Cranck-Nicolson scheme in time.

We introduce the approximation space $X_{h}$ with optimal approximation properties. The approximation space could either consist of finite element functions, with $X_{h} \subset C^{0}(\bar{\Omega})$, or approximation spaces with higher regularity such as NURBS, with $X_{h} \subset C^{1}(\bar{\Omega})$. In the finite element case, let $\mathcal{T}_{h}$ denote a conforming, shape-regular triangulation of $\Omega$ and let

$$
X_{h} \stackrel{\text { def }}{=}\left\{v_{h} \in H^{1}(\Omega):\left.v_{h}\right|_{K} \in P_{k}(K), \forall K \in \mathcal{T}_{h}\right\} .
$$

For the sake of simplicity (and in order to avoid technicalities) we assume that $\mathcal{T}_{h}$ is quasi-uniform. For NURBS with continuous derivatives, we refer the reader to [3] for a precise definition of the space and its properties.

We set $W_{h} \stackrel{\text { def }}{=}\left[X_{h}\right]^{d}$. The discrete spaces for velocities and pressure respectively are given by $V_{h} \stackrel{\text { def }}{=} W_{h} \cap V$ and $Q_{h} \stackrel{\text { def }}{=} X_{h} \cap Q$. The discrete time-derivative $\partial_{\tau, \kappa} \boldsymbol{u}_{h}^{n}$ is chosen either as the first-order $(\kappa=1)$

$$
\partial_{\tau, 1} \boldsymbol{u}_{h}^{n} \stackrel{\text { def }}{=}\left(\boldsymbol{u}_{h}^{n}-\boldsymbol{u}_{h}^{n-1}\right) / \tau,
$$


or second-order $(\kappa=2)$

$$
\partial_{\tau, 2} \boldsymbol{u}_{h}^{n} \stackrel{\text { def }}{=}\left(3 \boldsymbol{u}_{h}^{n}-4 \boldsymbol{u}_{h}^{n-1}+\boldsymbol{u}_{h}^{n-2}\right) /(2 \tau)
$$

backward difference formula. The formulation then reads: find $\left(\boldsymbol{u}_{h}^{n}, p_{h}^{n}\right) \in V_{h} \times$ $Q_{h}$ such that

$$
\left(\partial_{\tau, \kappa} \boldsymbol{u}_{h}^{n}+\boldsymbol{\nabla} p_{h}^{n}, \boldsymbol{w}_{h}\right)-\left(\nu \boldsymbol{\Delta} \boldsymbol{u}_{h}^{n}, \delta \boldsymbol{\nabla} q_{h}\right)_{h}+a\left(\boldsymbol{u}_{h}^{n}, \boldsymbol{v}_{h}\right)-b\left(q_{h}, \boldsymbol{u}_{h}^{n}\right)=\left(\boldsymbol{f}^{n}, \boldsymbol{w}_{h}\right)
$$

for all $\left(\boldsymbol{v}_{h}, q_{h}\right) \in V_{h} \times Q_{h}$ and where $\boldsymbol{w}_{h} \stackrel{\text { def }}{=} \boldsymbol{v}_{h}+\delta \boldsymbol{\nabla} q_{h}$. Here, $(\cdot, \cdot)_{h}$ denotes the element-wise $L^{2}$-scalar product and $\delta \stackrel{\text { def }}{=} \gamma h^{2} / \nu$, with $\gamma>0$ a dimensionless parameter.

The PSPG-method using the Crank-Nicolson method for the discretization in time takes the form: find $\left(\boldsymbol{u}_{h}^{n}, p_{h}^{n-\frac{1}{2}}\right) \in V_{h} \times Q_{h}$ such that

$$
\left(\partial_{\tau, 1} \boldsymbol{u}_{h}^{n}+\boldsymbol{\nabla} p_{h}^{n-\frac{1}{2}}, \boldsymbol{w}_{h}\right)-\left(\nu \boldsymbol{\Delta} \overline{\boldsymbol{u}}_{h}^{n}, \delta \boldsymbol{\nabla} q_{h}\right)_{h}+a\left(\overline{\boldsymbol{u}}_{h}^{n}, \boldsymbol{v}_{h}\right)-b\left(q_{h}, \overline{\boldsymbol{u}}_{h}^{n}\right)=\left(\boldsymbol{f}^{n-\frac{1}{2}}, \boldsymbol{w}_{h}\right)
$$

for all $\left(\boldsymbol{v}_{h}, q_{h}\right) \in V_{h} \times Q_{h}$ and where $\boldsymbol{w}_{h} \stackrel{\text { def }}{=} \boldsymbol{v}_{h}+\delta \boldsymbol{\nabla} q_{h}$. Here $\overline{\boldsymbol{u}}_{h}^{n} \stackrel{\text { def }}{=}\left(\boldsymbol{u}_{h}^{n}+\boldsymbol{u}_{h}^{n-1}\right) / 2$ denotes the average of $\boldsymbol{u}_{h}$ over the time-interval $\left(t^{n-1}, t^{n}\right)$. Note that $p_{h}^{n-\frac{1}{2}}$ is not defined by taking an average, but is an element in the space $Q_{h}$. Hence, at each time-step, the scheme provides an approximation $\left(\boldsymbol{u}_{h}^{n}, p_{h}^{n-\frac{1}{2}}\right)$ of $\left(\boldsymbol{u}\left(t^{n}\right), p\left(t^{n-\frac{1}{2}}\right)\right)$, that is, the velocity and pressure approximations are staggered in time by half a time-step.

For the convergence analysis below we introduce the following Ritz-projection: Find $\left(\boldsymbol{R}_{h}(\boldsymbol{u}, p), P_{h}(\boldsymbol{u}, p)\right) \in V_{h} \times Q_{h}$, such that

$$
\begin{array}{r}
a\left(\boldsymbol{R}_{h}(\boldsymbol{u}, p), \boldsymbol{v}_{h}\right)+b\left(\boldsymbol{R}_{h}(\boldsymbol{u}, p), \boldsymbol{v}_{h}\right)-b\left(q_{h}, \boldsymbol{R}_{h}(\boldsymbol{u}, p)\right) \\
+\left(-\boldsymbol{\Delta} \boldsymbol{R}_{h}(\boldsymbol{u}, p)+\boldsymbol{\nabla} P_{h}(\boldsymbol{u}, p), \delta \boldsymbol{\nabla} q_{h}\right)_{h}=a\left(\boldsymbol{u}, \boldsymbol{v}_{h}\right)+b\left(p, \boldsymbol{v}_{h}\right)-b\left(q_{h}, \boldsymbol{u}\right) \\
+\left(-\boldsymbol{\Delta u}+\boldsymbol{\nabla} p, \delta \boldsymbol{\nabla} q_{h}\right)
\end{array}
$$

for all $\left(\boldsymbol{v}_{h}, q_{h}\right) \in V_{h} \times Q_{h}$. It is known (see [19] in the finite element case and [3] for the NURBS case) that the above Ritz-projection satisfies the following a priori error estimate, for $j=0,1$ :

$$
\begin{array}{r}
\left\|\partial_{t}^{j}\left(\boldsymbol{R}_{h}(\boldsymbol{u}, p)-\boldsymbol{u}\right)\right\|_{H}+h\left(\left\|\partial_{t}^{j}\left(\boldsymbol{R}_{h}(\boldsymbol{u}, p)-\boldsymbol{u}\right)\right\|_{V}+\delta^{\frac{1}{2}}\left\|\partial_{t}^{j} \boldsymbol{\nabla}\left(P_{h}(\boldsymbol{u}, p)-p\right)\right\|_{H}\right. \\
\left.+\nu^{-\frac{1}{2}}\left\|\partial_{t}^{j}\left(P_{h}(\boldsymbol{u}, p)-p\right)\right\|_{H}\right) \leq C_{\nu} h^{k+1}\left(\left|\partial_{t}^{j} \boldsymbol{u}\right|_{k+1}+\left|\partial_{t}^{j} p\right|_{k}\right),
\end{array}
$$

where $C_{\nu}>0$ is a constant that depends on $\nu$.

For the stability analysis of affine pressure approximations, we shall also consider a reduced Ritz-projection $\left(\widetilde{\boldsymbol{R}}_{h} \boldsymbol{u}, \widetilde{P}_{h} \boldsymbol{u}\right) \in V_{h} \times Q_{h}$, obtained from (7) by omitting the term $\left(\boldsymbol{\Delta} \boldsymbol{u}, \delta \boldsymbol{\nabla} q_{h}\right)$ and taking $p=0$ in the right hand side, that is,

$$
a\left(\widetilde{\boldsymbol{R}}_{h} \boldsymbol{u}, \boldsymbol{v}_{h}\right)+b\left(\widetilde{\boldsymbol{R}}_{h} \boldsymbol{u}, \boldsymbol{v}_{h}\right)-b\left(q_{h}, \widetilde{\boldsymbol{R}}_{h} \boldsymbol{u}\right)-\left(\boldsymbol{\nabla} \widetilde{P}_{h} \boldsymbol{u}, \delta \boldsymbol{\nabla} q_{h}\right)_{h}=a\left(\boldsymbol{u}, \boldsymbol{v}_{h}\right)
$$

for all $\left(\boldsymbol{v}_{h}, q_{h}\right) \in V_{h} \times Q_{h}$. In this case the following stability estimate holds:

$$
\left\|\widetilde{\boldsymbol{R}}_{h} \boldsymbol{u}\right\|_{V}^{2}+\left\|\delta^{\frac{1}{2}} \nabla \widetilde{P}_{h} \boldsymbol{u}\right\|_{H}^{2} \lesssim\|\boldsymbol{u}\|_{V}^{2} .
$$




\section{Stability analysis}

This section is devoted to the energy-based stability analysis of (5) and (6). The velocity stability is analyzed in the next subsection. Subsection 4.2 presents the stability of the pressure approximations provided by (5) with $\kappa=1$.

\subsection{Velocity stability}

The stability analysis for the method (5) draws from earlier ideas applied to the SUPG method for the transient transport problem proposed in [8]. Since the mass-matrix is non-symmetric, global stability is obtained not by the standard choice of test functions, $\boldsymbol{v}_{h}=\boldsymbol{u}_{h}^{n}, q_{h}=p_{h}^{n}$, but with a perturbation added to the test function for the velocities. Indeed as we will see below stability is obtained by taking $\boldsymbol{v}_{h}=\boldsymbol{u}_{h}^{n}+\delta \partial_{\tau, \kappa} \boldsymbol{u}_{h}^{n}$

Lemma 4.1 (Backward differentiation schemes) Let $\left\{\left(\boldsymbol{u}_{h}^{n}, p_{h}^{n}\right)\right\}_{n=\kappa}^{N}$ denote the solution of (5). In the case $X_{h} \not \subset C^{1}(\bar{\Omega})$ and $k \geq 2$ we assume that $\delta \leq \tau^{\kappa}$. Then, for $\gamma$ small enough, there holds:

$$
\begin{aligned}
\left\|\boldsymbol{u}_{h}^{N}\right\|_{H}^{2}+\tau \sum_{n=\kappa}^{N}\left(\left\|\delta^{\frac{1}{2}}\left(\partial_{\tau, \kappa} \boldsymbol{u}_{h}^{n}+\boldsymbol{\nabla} p_{h}^{n}\right)\right\|_{H}^{2}+\left\|\boldsymbol{u}_{h}^{n}\right\|_{V}^{2}\right) & \\
& \lesssim\left(\delta+\frac{C_{\mathrm{P}}^{2}}{\nu}\right) \tau \sum_{n=\kappa}^{N}\left\|\boldsymbol{f}^{n}\right\|_{H}^{2}+\sum_{n=0}^{\kappa-1}\left(\left\|\boldsymbol{u}_{h}^{n}\right\|_{H}^{2}+\delta\left\|\boldsymbol{u}_{h}^{n}\right\|_{V}^{2}\right),
\end{aligned}
$$

where $C_{\mathrm{P}}$ denotes the constant of the Poincaré inequality.

Proof. First take $\boldsymbol{v}_{h}=\boldsymbol{u}_{h}^{n}$ and $q_{h}=p_{h}^{n}$, so that $\boldsymbol{w}_{h}=\boldsymbol{u}_{h}^{n}+\delta \boldsymbol{\nabla} p_{h}^{n}$ and

$$
\begin{array}{r}
\left(\partial_{\tau, \kappa} \boldsymbol{u}_{h}^{n}+\boldsymbol{\nabla} p_{h}^{n}, \boldsymbol{u}_{h}^{n}+\delta \boldsymbol{\nabla} p_{h}^{n}\right)-\delta\left(\nu \boldsymbol{\Delta} \boldsymbol{u}_{h}^{n}, \boldsymbol{\nabla} p_{h}^{n}\right)_{h}+\left(\boldsymbol{\nabla} \cdot \boldsymbol{u}_{h}^{n}, p_{h}^{n}\right)+\left\|\boldsymbol{u}_{h}^{n}\right\|_{V}^{2} \\
=\left(\boldsymbol{f}^{n}, \boldsymbol{u}_{h}^{n}+\delta \nabla p_{h}^{n}\right) .
\end{array}
$$

Now we test with $\boldsymbol{v}_{h}=\delta \partial_{\tau, \kappa} \boldsymbol{u}_{h}^{n}$ and $q_{h}=0$, which gives

$$
\left(\partial_{\tau, \kappa} \boldsymbol{u}_{h}^{n}+\boldsymbol{\nabla} p_{h}^{n}, \delta \partial_{\tau, \kappa} \boldsymbol{u}_{h}^{n}\right)+\delta\left(\nu \nabla \boldsymbol{u}_{h}^{n}, \boldsymbol{\nabla} \partial_{\tau, \kappa} \boldsymbol{u}_{h}^{n}\right)=\left(\boldsymbol{f}^{n}, \delta \partial_{\tau, \kappa} \boldsymbol{u}_{h}^{n}\right) .
$$

Summing the two equations yields, for $n \geq \kappa$,

$$
\begin{gathered}
\frac{1}{2 \kappa} \partial_{\tau, 1}\left\|\boldsymbol{u}_{h}^{n}\right\|_{H}^{2}+\frac{\kappa-1}{4}\left(\partial_{\tau, 1}\left\|\tilde{\boldsymbol{u}}_{h}^{n}\right\|_{H}^{2}+\tau^{-1}\left\|\boldsymbol{u}_{h}^{n}-\tilde{\boldsymbol{u}}_{h}^{n-1}\right\|_{H}^{2}\right)+\frac{2-\kappa}{2} \tau\left\|\partial_{\tau, 1} \boldsymbol{u}^{n}\right\|_{H}^{2} \\
+\left\|\boldsymbol{u}_{h}^{n}\right\|_{V}^{2}+\delta\left\|\partial_{\tau, \kappa} \boldsymbol{u}_{h}^{n}+\nabla p_{h}^{n}\right\|_{H}^{2} \underbrace{-\delta\left(\nu \boldsymbol{\Delta} \boldsymbol{u}_{h}^{n}, \boldsymbol{\nabla} p_{h}^{n}\right)_{h}+\delta\left(\nu \boldsymbol{\nabla} \boldsymbol{u}_{h}^{n}, \boldsymbol{\nabla} \partial_{\tau, \kappa} \boldsymbol{u}_{h}^{n}\right)}_{I_{1}} \\
=\left(\boldsymbol{f}^{n}, \boldsymbol{u}_{h}^{n}+\delta\left(\partial_{\tau, \kappa} \boldsymbol{u}_{h}^{n}+\boldsymbol{\nabla} p_{h}^{n}\right)\right) .
\end{gathered}
$$

Where the notation $\tilde{\boldsymbol{u}}_{h}^{n}=2 \boldsymbol{u}_{h}^{n}-\boldsymbol{u}_{h}^{n-1}$ is used in the contribution from the BDF2-scheme and

$$
\partial_{\tau, 1}\left\|\boldsymbol{u}_{h}^{n}\right\|_{H}^{2} \stackrel{\text { def }}{=} \tau^{-1}\left(\left\|\boldsymbol{u}_{h}^{n}\right\|_{H}^{2}-\left\|\boldsymbol{u}_{h}^{n-1}\right\|_{H}^{2}\right) .
$$

For piecewise linear approximations $\left.\boldsymbol{\Delta} \boldsymbol{u}_{h}^{n}\right|_{K}=0$ and, hence, we have

$$
I_{1} \geq \frac{\delta}{2 \kappa} \partial_{\tau, 1}\left\|\boldsymbol{u}_{h}^{n}\right\|_{V}^{2}+\frac{\kappa-1}{4} \delta \partial_{\tau, 1}\left\|\tilde{\boldsymbol{u}}_{h}^{n}\right\|_{V}^{2},
$$


which telescopes by summing over $n=\kappa, \ldots, N$. When $\left.\boldsymbol{\Delta} \boldsymbol{u}_{h}^{n}\right|_{K} \neq 0$, the term $I_{1}$ needs a more specific treatment. Note that the following holds

$$
I_{1}=-\delta\left(\nu \boldsymbol{\Delta} \boldsymbol{u}_{h}^{n}, \partial_{\tau, \kappa} \boldsymbol{u}_{h}^{n}+\boldsymbol{\nabla} p_{h}^{n}\right)_{h}+\delta\left(\nu \boldsymbol{\Delta} \boldsymbol{u}_{h}^{n}, \partial_{\tau, \kappa} \boldsymbol{u}_{h}^{n}\right)_{h}+\delta\left(\nu \boldsymbol{\nabla} \boldsymbol{u}_{h}^{n}, \boldsymbol{\nabla} \partial_{\tau, \kappa} \boldsymbol{u}_{h}^{n}\right)
$$

and, after an integration by parts in the second term of the right-hand side, we get

$$
I_{1}=-\underbrace{\delta\left(\nu \boldsymbol{\Delta} \boldsymbol{u}_{h}^{n}, \partial_{\tau, \kappa} \boldsymbol{u}_{h}^{n}+\nabla p_{h}^{n}\right)_{h}}_{I_{2}}-\underbrace{\sum_{F \in \mathcal{F}_{\mathrm{i}}} \delta \nu \int_{F} \llbracket \boldsymbol{n} \cdot \boldsymbol{\nabla} \boldsymbol{u}_{h}^{n} \rrbracket \cdot \partial_{\tau, \kappa} \boldsymbol{u}_{h}^{n}}_{I_{3}},
$$

where $\mathcal{F}_{\mathrm{i}}$ stands for the set of interior faces $F$ of $\mathcal{T}_{h}$ and $\llbracket \boldsymbol{n} \cdot \boldsymbol{\nabla} \boldsymbol{u}_{h}^{n} \rrbracket$ denotes the component-wise jump of the vector $\boldsymbol{n} \cdot \boldsymbol{\nabla} \boldsymbol{u}_{h}^{n}$. For the first term in the right-hand side of (12) we may use the Cauchy-Schwarz inequality, an inverse inequality and the arithmetic-geometric inequality to write

$$
I_{2} \geq-\frac{C_{\mathrm{inv}}^{2} \gamma^{\frac{1}{2}}}{4 \epsilon}\left\|\boldsymbol{u}_{h}^{n}\right\|_{V}^{2}-\epsilon\left\|\delta^{\frac{1}{2}}\left(\partial_{\tau, \kappa} \boldsymbol{u}_{h}^{n}+\nabla p_{h}^{n}\right)\right\|_{H}^{2} .
$$

Here (and below) $C_{\mathrm{inv}}$ denotes the constant in the standard inverse inequality $\left\|\boldsymbol{\nabla} \boldsymbol{u}_{h}^{n}\right\|_{0, \Omega} \leq C_{\text {inv }} h^{-1}\left\|\boldsymbol{u}_{h}^{n}\right\|_{0, \Omega}$. Choosing $\epsilon$ and $\gamma$ small enough we see that the term can be absorbed in the left-hand side of 100 . The term that remains to control is hence $I_{3}$. For $C^{1}(\bar{\Omega})$ approximation spaces this term is zero. Hence, for piece-wise linear approximations $\left(I_{1}=0\right)$ and $C^{1}$ NURBS we conclude by multiplying (10) with $\tau$, summing over the time levels and applying the Cauchy-Schwarz inequality, the arithmetic-geometric inequality and the Poincaré inequality $\left\|\boldsymbol{u}_{h}\right\|_{H} \leq C_{\mathrm{P}}\left\|\boldsymbol{\nabla} \boldsymbol{u}_{h}\right\|_{H}$ in the right hand side:

$$
\begin{aligned}
\left|\left(\boldsymbol{f}^{n}, \boldsymbol{u}_{h}^{n}+\delta\left(\partial_{\tau, \kappa} \boldsymbol{u}_{h}^{n}+\nabla p_{h}^{n}\right)\right)\right| \leq\left(\delta+\frac{C_{\mathrm{P}}^{2}}{\nu}\right) & \left\|\boldsymbol{f}^{n}\right\|_{H}^{2}+\frac{1}{4}\left\|\boldsymbol{u}_{h}^{n}\right\|_{V}^{2} \\
& +\frac{1}{4}\left\|\delta^{\frac{1}{2}}\left(\partial_{\tau, \kappa} \boldsymbol{u}_{h}^{n}+\nabla p_{h}^{n}\right)\right\|_{H}^{2} .
\end{aligned}
$$

The resulting contributions $\left\|\delta^{\frac{1}{2}}\left(\partial_{\tau, \kappa} \boldsymbol{u}_{h}^{n}+\nabla p_{h}^{n}\right)\right\|_{H}^{2}$ and $\left\|\boldsymbol{u}_{h}\right\|_{V}^{2}$ may be absorbed in the left hand side of (10).

In the case of high-order finite element approximation, i.e. $k \geq 2$, different approaches must be used for $\kappa=1$ and $\kappa=2$. In both cases we start with the following bound on (11), obtained by applying the Cauchy-Schwarz inequality followed by an inverse inequality,

$$
\begin{aligned}
I_{1} \geq & -\frac{3 C_{\mathrm{inv}}^{2} \gamma}{2}\left\|\boldsymbol{u}_{h}^{n}\right\|_{V}^{2}-\frac{1}{4}\left\|\delta^{\frac{1}{2}}\left(\partial_{\tau, \kappa} \boldsymbol{u}_{h}^{n}+\nabla p_{h}^{n}\right)\right\|_{H}^{2}-\frac{\delta}{2}\left\|\partial_{\tau, \kappa} \boldsymbol{u}_{h}^{n}\right\|_{H}^{2} \\
& +\frac{\delta}{2 \kappa} \partial_{\tau, 1}\left\|\boldsymbol{u}_{h}^{n}\right\|_{V}^{2}+\frac{\kappa-1}{4} \delta \partial_{\tau, 1}\left\|\tilde{\boldsymbol{u}}_{h}^{n}\right\|_{V}^{2} .
\end{aligned}
$$

For BDF1 $(\kappa=1)$, under the inverse CFL-condition $\delta \leq \tau$, we may absorb the term $\frac{1}{2} \delta\left\|\partial_{\tau, 1} \boldsymbol{u}_{h}^{n}\right\|_{H}^{2}$ using the dissipative contribution $\frac{1}{2} \tau\left\|\partial_{\tau, 1} \boldsymbol{u}_{h}^{n}\right\|_{H}^{2}$ in the left hand side of 10. Assuming $\gamma$ small enough, this leads to the estimate

$\partial_{\tau, 1}\left\|\boldsymbol{u}_{h}^{n}\right\|_{H}^{2}+\left\|\delta^{\frac{1}{2}}\left(\partial_{\tau, 1} \boldsymbol{u}_{h}^{n}+\nabla p_{h}^{n}\right)\right\|_{H}^{2}+\left\|\boldsymbol{u}_{h}^{n}\right\|_{V}^{2}+\delta \partial_{\tau, 1}\left\|\boldsymbol{u}_{h}^{n}\right\|_{V}^{2} \lesssim\left(\delta+\frac{C_{\mathrm{P}}^{2}}{\nu}\right)\left\|\boldsymbol{f}^{n}\right\|_{H}^{2}$. 
For BDF2 $(\kappa=2)$, the term $\frac{1}{2} \tau\left\|\partial_{\tau, \kappa} \boldsymbol{u}_{h}^{n}\right\|_{H}^{2}$ is not present in the left hand side of (10) and the dissipation resulting from the BDF2 scheme, $\frac{1}{4} \tau^{-1}\left\|\boldsymbol{u}_{h}^{n}-\tilde{\boldsymbol{u}}_{h}^{n-1}\right\|_{H}^{2}$, may not be used to obtain stability. In this case we assume $\delta^{\frac{1}{2}} \leq \tau$, so that the third term in the right-hand side of (14) may be bounded as

$$
\delta\left\|\partial_{\tau, 2} \boldsymbol{u}_{h}^{n}\right\|_{H}^{2} \lesssim \gamma \sum_{j=0}^{2}\left\|\boldsymbol{u}_{h}^{n-j}\right\|_{H}^{2} .
$$

For $\gamma$ small enough we then obtain the stability estimate

$$
\begin{aligned}
\partial_{\tau, 1}\left\|\boldsymbol{u}^{n}\right\|_{H}^{2}+\partial_{\tau, 1}\left\|\tilde{\boldsymbol{u}}_{h}^{n}\right\|_{H}^{2}+\left\|\delta^{\frac{1}{2}}\left(\partial_{\tau, \kappa} \boldsymbol{u}_{h}^{n}+\nabla p_{h}^{n}\right)\right\|_{H}^{2}+\left\|\boldsymbol{u}_{h}^{n}\right\|_{V}^{2} \\
\quad+\delta \partial_{\tau, 1}\left(\left\|\boldsymbol{u}_{h}^{n}\right\|_{V}^{2}+\left\|\tilde{\boldsymbol{u}}_{h}^{n}\right\|_{V}^{2}\right) \lesssim\left(\delta+\frac{C_{\mathrm{P}}^{2}}{\nu}\right)\left\|\boldsymbol{f}^{n}\right\|_{H}^{2}+\gamma \sum_{j=0}^{2}\left\|\boldsymbol{u}_{h}^{n-j}\right\|_{H}^{2} .
\end{aligned}
$$

We multiply the equations (15) and with $\tau$ and sum over $n=\kappa, \ldots, N$ to conclude. For BDF2 we apply Gronwall's lemma (also requiring $\gamma$ small enough). $\diamond$

Remark 4.2 Note that the stability condition reported in Lemma 4.1 comes from the non-conformity of the Laplacian operator approximation, which demands a specific treatment of the term $\delta \nu\left[\left(-\boldsymbol{\Delta} \boldsymbol{u}_{h}^{n}, \partial_{\tau, \kappa} \boldsymbol{u}_{h}^{n}\right)_{h}+\left(\boldsymbol{\nabla} \boldsymbol{u}_{h}^{n}, \boldsymbol{\nabla} \partial_{\tau, \kappa} \boldsymbol{u}_{h}^{n}\right)\right]$ in (11). This issue will be addressed in section 6 , using an alternative formulation of the PSPG method (5).

The analysis of the Crank-Nicolson time-discretization is similar and actually simpler. We include it here for the readers convenience and for further reference in the numerical section.

Lemma 4.3 (Crank-Nicolson scheme) Let $\left\{\left(\boldsymbol{u}_{h}^{n}, p_{h}^{n-\frac{1}{2}}\right)\right\}_{n=1}^{N}$ be given by (6). In the case $X_{h} \not \subset C^{1}(\bar{\Omega})$ and $k \geq 2$ we assume that $\delta \lesssim \tau^{2}$. Then, for $\gamma$ sufficiently small, there holds:

$$
\begin{aligned}
\left\|\boldsymbol{u}_{h}^{N}\right\|_{H}^{2}+\tau \sum_{n=1}^{N}\left(\left\|\delta^{\frac{1}{2}}\left(\partial_{\tau, 1} \boldsymbol{u}_{h}^{n}+\nabla p_{h}^{n-\frac{1}{2}}\right)\right\|_{H}^{2}+\left\|\overline{\boldsymbol{u}}_{h}^{n}\right\|_{V}^{2}\right) & \\
& \lesssim\left(\delta+\frac{C_{\mathrm{P}}^{2}}{\nu}\right) \tau \sum_{n=1}^{N}\left\|\boldsymbol{f}^{n-\frac{1}{2}}\right\|_{H}^{2}+\left\|\boldsymbol{u}_{h}^{0}\right\|_{H}^{2}+\delta\left\|\boldsymbol{u}_{h}^{0}\right\|_{V}^{2} .
\end{aligned}
$$

Proof. First we take $\boldsymbol{v}_{h}=\overline{\boldsymbol{u}}_{h}^{n}$ and $q_{h}=p_{h}^{n-\frac{1}{2}}$ in (6), so that $\boldsymbol{w}_{h}=\overline{\boldsymbol{u}}_{h}^{n}+$ $\delta \nabla p_{h}^{n-\frac{1}{2}}$ and

$$
\begin{aligned}
\left(\partial_{\tau, 1} \boldsymbol{u}_{h}^{n}+\nabla p_{h}^{n-\frac{1}{2}}, \overline{\boldsymbol{u}}_{h}^{n}+\delta \boldsymbol{\nabla} p_{h}^{n-\frac{1}{2}}\right) & -\delta\left(\nu \boldsymbol{\Delta} \overline{\boldsymbol{u}}_{h}^{n}, \boldsymbol{\nabla} p_{h}^{n-\frac{1}{2}}\right)_{h}+\left\|\overline{\boldsymbol{u}}_{h}^{n}\right\|_{V}^{2} \\
& +\left(\nabla \cdot \overline{\boldsymbol{u}}_{h}^{n}, p_{h}^{n-\frac{1}{2}}\right)=\left(\boldsymbol{f}^{n-\frac{1}{2}}, \overline{\boldsymbol{u}}_{h}^{n}+\delta \boldsymbol{\nabla} p_{h}^{n-\frac{1}{2}}\right)
\end{aligned}
$$

Now we test (6) with $\boldsymbol{v}_{h}=\delta \partial_{\tau, 1} \boldsymbol{u}_{h}^{n}$ and $q_{h}=0$, yielding

$$
\left(\partial_{\tau, 1} \boldsymbol{u}_{h}^{n}+\nabla p_{h}^{n-\frac{1}{2}}, \delta \partial_{\tau, 1} \boldsymbol{u}_{h}^{n}\right)+\delta\left(\nu \nabla \overline{\boldsymbol{u}}_{h}^{n}, \nabla \partial_{\tau, 1} \boldsymbol{u}_{h}^{n}\right)=\left(\boldsymbol{f}^{n-\frac{1}{2}}, \delta \partial_{\tau, 1} \boldsymbol{u}_{h}^{n}\right)
$$


Summing the two equalities yields, for $n \geq 1$,

$$
\begin{aligned}
& \frac{1}{2} \partial_{\tau, 1}\left\|\boldsymbol{u}_{h}^{n}\right\|_{H}^{2}+\left\|\overline{\boldsymbol{u}}_{h}^{n}\right\|_{V}^{2}+\left\|\delta^{\frac{1}{2}}\left(\partial_{\tau, 1} \boldsymbol{u}_{h}^{n}+\boldsymbol{\nabla} p_{h}^{n-\frac{1}{2}}\right)\right\|_{H}^{2} \\
& \underbrace{-\delta\left(\nu \boldsymbol{\Delta} \overline{\boldsymbol{u}}_{h}^{n}, \boldsymbol{\nabla} p_{h}^{n-\frac{1}{2}}\right)_{h}+\delta\left(\nu \boldsymbol{\nabla} \overline{\boldsymbol{u}}_{h}^{n}, \boldsymbol{\nabla} \partial_{\tau, 1} \boldsymbol{u}_{h}^{n}\right)}_{I_{1}} \\
&=\left(\boldsymbol{f}^{n-\frac{1}{2}}, \overline{\boldsymbol{u}}_{h}^{n}+\delta\left(\partial_{\tau, 1} \boldsymbol{u}_{h}^{n}+\boldsymbol{\nabla} p_{h}^{n-\frac{1}{2}}\right)\right) .
\end{aligned}
$$

Now we proceed as in the proof of Lemma 4.1. For piecewise linear approximations $\left(\left.\boldsymbol{\Delta} \boldsymbol{u}_{h}^{n}\right|_{K}=0\right)$ we have $I_{1}=\frac{1}{2} \delta \partial_{\tau, 1}\left\|\boldsymbol{u}_{h}^{n}\right\|_{V}^{2}$, which telescopes when summing over $n=1, \ldots, N$. In the general case $\left(\left.\boldsymbol{\Delta} \boldsymbol{u}_{h}^{n}\right|_{K} \neq 0\right)$, we observe that $I_{1}=-\delta\left(\nu \boldsymbol{\Delta} \overline{\boldsymbol{u}}_{h}^{n}, \partial_{\tau, 1} \boldsymbol{u}_{h}^{n}+\boldsymbol{\nabla} p_{h}^{n-\frac{1}{2}}\right)_{h}+\delta\left(\nu \boldsymbol{\Delta} \overline{\boldsymbol{u}}_{h}^{n}, \partial_{\tau, 1} \boldsymbol{u}_{h}^{n}\right)_{h}+\delta\left(\nu \nabla \overline{\boldsymbol{u}}_{h}^{n}, \boldsymbol{\nabla} \partial_{\tau, 1} \boldsymbol{u}_{h}^{n}\right)$, so that, after an integration by parts in the second term in the right-hand side, we get

$$
I_{1}=-\underbrace{\delta\left(\nu \boldsymbol{\Delta} \overline{\boldsymbol{u}}_{h}^{n}, \partial_{\tau, 1} \boldsymbol{u}_{h}^{n}+\nabla p_{h}^{n-\frac{1}{2}}\right)_{h}}_{I_{2}}-\underbrace{\delta \nu \sum_{F} \int_{F \in \mathcal{F}_{i}} \llbracket \boldsymbol{n} \cdot \nabla \overline{\boldsymbol{u}}_{h}^{n} \rrbracket \cdot \partial_{\tau, 1} \boldsymbol{u}_{h}^{n}}_{I_{3}},
$$

which is the Crank-Nicholson counterpart of 12 . Proceeding as in the proof of Lemma 4.1, we have

$$
I_{2} \geq-\frac{C_{\mathrm{inv}}^{2} \gamma^{\frac{1}{2}}}{4 \epsilon}\left\|\overline{\boldsymbol{u}}_{h}^{n}\right\|_{V}^{2}-\epsilon\left\|\delta^{\frac{1}{2}}\left(\partial_{\tau, 1} \boldsymbol{u}_{h}^{n}+\nabla p_{h}^{n-\frac{1}{2}}\right)\right\|_{H}^{2}
$$

and this term can be absorbed in the left-hand side of (17) by choosing $\epsilon$ and $\gamma$ small enough. Once more, for $C^{1}$-approximation spaces the term $I_{3}$ vanishes. Hence, for piecewise linear approximations $\left(I_{1}=0\right)$ and $C^{1}$ NURBS we conclude as in the proof of Lemma 4.1 by noting that

$$
\begin{aligned}
\left|\left(\boldsymbol{f}^{n-\frac{1}{2}}, \overline{\boldsymbol{u}}_{h}^{n}+\delta\left(\partial_{\tau, 1} \boldsymbol{u}_{h}^{n}+\boldsymbol{\nabla} p_{h}^{n-\frac{1}{2}}\right)\right)\right| \leq(\delta+ & \left.\frac{C_{\mathrm{P}}^{2}}{\nu}\right)\left\|\boldsymbol{f}^{n-\frac{1}{2}}\right\|_{H}^{2}+\frac{1}{4}\left\|\overline{\boldsymbol{u}}_{h}^{n}\right\|_{V}^{2} \\
& +\frac{1}{4}\left\|\delta^{\frac{1}{2}}\left(\partial_{\tau, 1} \boldsymbol{u}_{h}^{n}+\boldsymbol{\nabla} p_{h}^{n-\frac{1}{2}}\right)\right\|_{H}^{2} .
\end{aligned}
$$

For high-order finite element approximations $(k \geq 2)$, the term $I_{1}$ is bounded as in (14), yielding

$$
I_{1} \geq-\frac{5 C_{\text {inv }}^{2} \gamma}{4}\left\|\overline{\boldsymbol{u}}_{h}^{n}\right\|_{V}^{2}-\frac{1}{4}\left\|\delta^{\frac{1}{2}}\left(\partial_{\tau, 1} \boldsymbol{u}_{h}^{n}+\nabla p_{h}^{n}\right)\right\|_{H}^{2}-\delta\left\|\partial_{\tau, 1} \boldsymbol{u}_{h}^{n}\right\|_{H}^{2}+\frac{\delta}{2} \partial_{\tau, 1}\left\|\boldsymbol{u}_{h}^{n}\right\|_{V}^{2} .
$$

On the other hand, from the assumption $\delta^{\frac{1}{2}} \lesssim \tau$ we have $\delta\left\|\partial_{\tau, 1} \boldsymbol{u}_{h}^{n}\right\|_{H}^{2} \lesssim$ $\gamma\left(\left\|\boldsymbol{u}_{h}^{n}\right\|_{H}^{2}+\left\|\boldsymbol{u}_{h}^{n-1}\right\|_{H}^{2}\right)$, so that

$$
\begin{aligned}
\partial_{\tau, 1}\left\|\boldsymbol{u}^{n}\right\|_{H}^{2}+\| \delta^{\frac{1}{2}}\left(\partial_{\tau, \kappa} \boldsymbol{u}_{h}^{n}+\right. & \left.\nabla p_{h}^{n-\frac{1}{2}}\right)\left\|_{H}^{2}+\right\| \overline{\boldsymbol{u}}_{h}^{n}\left\|_{V}^{2}+\delta \partial_{\tau, 1}\right\| \boldsymbol{u}_{h}^{n} \|_{V}^{2} \\
& \lesssim\left(\delta+\frac{C_{\mathrm{P}}^{2}}{\nu}\right)\left\|\boldsymbol{f}^{n-\frac{1}{2}}\right\|_{H}^{2}+\gamma\left(\left\|\boldsymbol{u}_{h}^{n}\right\|_{H}^{2}+\left\|\boldsymbol{u}_{h}^{n-1}\right\|_{H}^{2}\right)
\end{aligned}
$$

for $\gamma>0$ small enough. We conclude after multiplication by $\tau$, summation over $n=1, \ldots, N$ and the application of Gronwall's lemma (also requiring $\gamma$ small enough). $\diamond$ 


\subsection{Pressure stability}

For the pressure stability analysis below, we shall make use of the following modified inf-sup condition.

Lemma 4.4 For all $q_{h} \in Q_{h}$ and $\boldsymbol{\xi}_{h} \in V_{h}$ we have

$$
\left\|q_{h}\right\|_{Q} \lesssim \sup _{\boldsymbol{v}_{h} \in V_{h}} \frac{\left|\left(q_{h}, \boldsymbol{\nabla} \cdot \boldsymbol{v}_{h}\right)\right|}{\left\|\boldsymbol{v}_{h}\right\|_{V}}+\gamma^{-\frac{1}{2}}\left\|\delta^{\frac{1}{2}}\left(\boldsymbol{\nabla} q_{h}+\boldsymbol{\xi}_{h}\right)\right\|_{H} .
$$

Proof. Let $q_{h} \in Q_{h}$, it is known (see, e.g., [14, Corollary 2.4]) that there exists $\boldsymbol{v}_{q} \in\left[H_{0}^{1}(\Omega)\right]^{d}$ such that $\boldsymbol{\nabla} \cdot \boldsymbol{v}_{q}=-\nu^{-1} q_{h}$ and $\left\|\boldsymbol{v}_{q}\right\|_{V} \lesssim\left\|q_{h}\right\|_{Q}$. Hence, from the orthogonality and approximation properties of the $L^{2}$-projection $\pi_{h}$, onto $V_{h}$, for $\boldsymbol{\xi}_{h} \in V_{h}$ we have

$$
\begin{aligned}
\left\|q_{h}\right\|_{Q}^{2} & =-\left(q_{h}, \boldsymbol{\nabla} \cdot\left(\boldsymbol{v}_{q}-\pi_{h} \boldsymbol{v}_{q}\right)\right)-\left(q_{h}, \boldsymbol{\nabla} \cdot \pi_{h} \boldsymbol{v}_{q}\right) \\
& =\left(\boldsymbol{\nabla} q_{h}+\boldsymbol{\xi}_{h}, \boldsymbol{v}_{q}-\pi_{h} \boldsymbol{v}_{q}\right)-\left(q_{h}, \boldsymbol{\nabla} \cdot \pi_{h} \boldsymbol{v}_{q}\right) \\
& \lesssim \gamma^{-\frac{1}{2}}\left\|\delta^{\frac{1}{2}}\left(\boldsymbol{\nabla} q_{h}+\boldsymbol{\xi}_{h}\right)\right\|_{H}\left\|q_{h}\right\|_{Q}+\left|\left(q_{h}, \boldsymbol{\nabla} \cdot \pi_{h} \boldsymbol{v}_{q}\right)\right|
\end{aligned}
$$

which completes the proof by noting that $\left\|\pi_{h} \boldsymbol{v}_{q}\right\|_{V} \lesssim\left\|\boldsymbol{v}_{q}\right\|_{V}$ for quasi-uniform triangulations (see [12]).

The next theorem states the stability of affine PSPG pressure approximations with the BDF1 scheme, in the natural discrete $L^{2}\left(0, T ; L^{2}(\Omega)\right)$-norm, under the inverse parabolic CFL-condition $\delta \lesssim \tau$.

Theorem 4.5 Assume that $\boldsymbol{f} \in C^{1}(0, T ; H)$ and let $\left\{\left(\boldsymbol{u}_{h}^{n}, p_{h}^{n}\right)\right\}_{n=1}^{N}$ be given by (5) with $\kappa=1$ and $k=1$ in (4).

- Assume that $\boldsymbol{u}_{0} \in\left[H^{2}(\Omega)\right]^{d}$ and let $\boldsymbol{u}_{h}^{0}=\mathcal{I}_{h} \boldsymbol{u}_{0}$, where $\mathcal{I}_{h}$ is the Lagrange interpolation operator onto $V_{h}$. Then we have

$$
\begin{aligned}
\tau\left(1-\frac{C_{\mathcal{I}}^{2} \delta}{\gamma \tau}\right)\left\|p_{h}^{1}\right\|_{Q}^{2}+\tau & \sum_{n=2}^{N}\left\|p_{h}^{n}\right\|_{Q}^{2} \lesssim C_{\mathrm{P}}^{2}\left(1+\frac{\delta}{\tau}\right)\left\|\nabla \boldsymbol{u}_{h}^{0}\right\|_{H}^{2}+\frac{C_{\mathrm{P}}^{2}}{\nu}\left\|\delta^{\frac{1}{2}} \boldsymbol{f}^{1}\right\|_{H}^{2} \\
+C_{\mathrm{P}}^{4}\left|\boldsymbol{u}_{0}\right|_{2, \Omega}^{2}+ & \tau \sum_{n=1}^{N}\left(\left\|\boldsymbol{u}_{h}^{n}\right\|_{V}^{2}+\left\|\delta^{\frac{1}{2}}\left(\boldsymbol{\nabla} p_{h}^{n}+\partial_{\tau, 1} \boldsymbol{u}_{h}^{n}\right)\right\|_{H}^{2}\right) \\
& +\frac{C_{\mathrm{P}}^{2} \tau}{\nu} \sum_{n=1}^{N}\left(\frac{C_{\mathrm{P}}^{2}}{\nu}\left\|\delta^{\frac{1}{2}} \partial_{\tau, 1} \boldsymbol{f}^{n}\right\|_{H}^{2}+\left\|\boldsymbol{f}^{n}\right\|_{H}^{2}\right)
\end{aligned}
$$

- Assume that $\boldsymbol{u}_{0} \in\left[H^{1}(\Omega)\right]^{d}$ and let $\boldsymbol{u}_{h}^{0}=\widetilde{\boldsymbol{R}}_{h} \boldsymbol{u}_{0}$. Then we have

$$
\begin{aligned}
\tau \sum_{n=1}^{N}\left\|p_{h}^{n}\right\|_{Q}^{2} \lesssim & C_{\mathrm{P}}^{2}\left(1+\frac{\delta}{\tau}\right)\left\|\nabla \boldsymbol{u}_{h}^{0}\right\|_{H}^{2}+\frac{C_{\mathrm{P}}^{2}}{\nu}\left\|\delta^{\frac{1}{2}} \boldsymbol{f}^{1}\right\|_{H}^{2} \\
+ & +\sum_{n=1}^{N}\left(\left\|\boldsymbol{u}_{h}^{n}\right\|_{V}^{2}+\left\|\delta^{\frac{1}{2}}\left(\boldsymbol{\nabla} p_{h}^{n}+\partial_{\tau, 1} \boldsymbol{u}_{h}^{n}\right)\right\|_{H}^{2}\right) \\
& +\frac{C_{\mathrm{P}}^{2} \tau}{\nu} \sum_{n=1}^{N}\left(\frac{C_{\mathrm{P}}^{2}}{\nu}\left\|\delta^{\frac{1}{2}} \partial_{\tau, 1} \boldsymbol{f}^{n}\right\|_{H}^{2}+\left\|\boldsymbol{f}^{n}\right\|_{H}^{2}\right) .
\end{aligned}
$$


Proof. From (19) and (5), we have

$\tau \sum_{n=1}^{N}\left\|p_{h}^{n}\right\|_{Q}^{2} \lesssim \tau \sum_{n=1}^{N}\left(\left\|\delta^{\frac{1}{2}}\left(\nabla p_{h}^{n}+\partial_{\tau, 1} \boldsymbol{u}_{h}^{n}\right)\right\|_{H}^{2}+\frac{C_{\mathrm{P}}^{2}}{\nu}\left\|\partial_{\tau, 1} \boldsymbol{u}_{h}^{n}\right\|_{H}^{2}+\left\|\boldsymbol{u}_{h}^{n}\right\|_{V}^{2}+\frac{C_{\mathrm{P}}^{2}}{\nu}\left\|\boldsymbol{f}^{n}\right\|_{H}^{2}\right)$.

As a result, we only need to estimate the acceleration

$$
\frac{C_{\mathrm{P}}^{2} \tau}{\nu} \sum_{n=1}^{N}\left\|\partial_{\tau, 1} \boldsymbol{u}_{h}^{n}\right\|_{H}^{2}
$$

To this aim, we test (5) with $\boldsymbol{v}_{h}=C_{\mathrm{P}}^{2} \nu^{-1} \tau \partial_{\tau, 1} \boldsymbol{u}_{h}^{n}$ and $q_{h}=0$, multiply by $2 \tau$ and sum over $n=1, \ldots, N$. This yields

$$
\begin{aligned}
\frac{C_{\mathrm{P}}^{2} \tau}{\nu} \sum_{n=1}^{N}\left\|\partial_{\tau, 1} \boldsymbol{u}_{h}^{n}\right\|_{H}^{2}+C_{\mathrm{P}}^{2}\left\|\boldsymbol{\nabla} \boldsymbol{u}_{h}^{N}\right\|_{H}^{2} & -2 \frac{C_{\mathrm{P}}^{2} \tau}{\nu} \sum_{n=1}^{N}\left(p_{h}^{n}, \nabla \cdot \partial_{\tau, 1} \boldsymbol{u}_{h}^{n}\right) \\
& \leq C_{\mathrm{P}}^{2}\left\|\boldsymbol{\nabla} \boldsymbol{u}_{h}^{0}\right\|_{H}^{2}+\frac{C_{\mathrm{P}}^{2} \tau}{\nu} \sum_{n=1}^{N}\left\|\boldsymbol{f}^{n}\right\|_{H}^{2} .
\end{aligned}
$$

We must now show that the term

$$
-\frac{C_{\mathrm{P}}^{2} \tau}{\nu} \sum_{n=1}^{N}\left(p_{h}^{n}, \nabla \cdot \partial_{\tau, 1} \boldsymbol{u}_{h}^{n}\right)=\underbrace{-\frac{C_{\mathrm{P}}^{2} \tau}{\nu} \sum_{n=2}^{N}\left(p_{h}^{n}, \nabla \cdot \partial_{\tau, 1} \boldsymbol{u}_{h}^{n}\right)}_{I_{1}} \underbrace{-\frac{C_{\mathrm{P}}^{2} \tau}{\nu}\left(p_{h}^{1}, \nabla \cdot \partial_{\tau, 1} \boldsymbol{u}_{h}^{n}\right)}_{I_{2}}
$$

can be appropriately bounded. From (5) with $\boldsymbol{v}_{h}=0$, for $n \geq 2$ we have

$$
-\left(q_{h}, \nabla \cdot \partial_{\tau, 1} \boldsymbol{u}_{h}^{n}\right)=\left(\partial_{\tau, 1}\left(\partial_{\tau, 1} \boldsymbol{u}_{h}^{n}+\nabla p_{h}^{n}-\boldsymbol{f}^{n}\right), \delta \nabla q_{h}\right) .
$$

Hence, taking $q_{h}=\nu^{-1} \tau p_{h}^{n}$ and after summation over $n=2, \ldots, N$, we get

$$
\begin{gathered}
I_{1}=\underbrace{\frac{C_{\mathrm{P}}^{2} \tau}{\nu} \sum_{n=2}^{N}\left(\partial_{\tau, 1}\left(\partial_{\tau, 1} \boldsymbol{u}_{h}^{n}+\nabla p_{h}^{n}-\boldsymbol{f}^{n}\right), \delta\left(\partial_{\tau, 1} \boldsymbol{u}_{h}^{n}+\nabla p_{h}^{n}\right)\right)}_{I_{3}} \\
\\
\underbrace{-\frac{C_{\mathrm{P}}^{2} \tau}{\nu} \sum_{n=2}^{N}\left(\partial_{\tau, 1}\left(\partial_{\tau, 1} \boldsymbol{u}_{h}^{n}+\nabla p_{h}^{n}-\boldsymbol{f}^{n}\right), \delta \partial_{\tau, 1} \boldsymbol{u}_{h}^{n}\right)}_{I_{4}} .
\end{gathered}
$$


Term $I_{3}$ can be lower bounded as follows:

$$
\begin{aligned}
I_{3}= & \frac{C_{\mathrm{P}}^{2} \tau}{\nu} \sum_{n=2}^{N}\left(\partial_{\tau, 1}\left(\partial_{\tau, 1} \boldsymbol{u}_{h}^{n}+\nabla p_{h}^{n}\right), \delta\left(\partial_{\tau, 1} \boldsymbol{u}_{h}^{n}+\nabla p_{h}^{n}\right)\right) \\
& -\frac{C_{\mathrm{P}}^{2} \tau}{\nu} \sum_{n=2}^{N}\left(\partial_{\tau, 1} \boldsymbol{f}^{n}, \delta\left(\partial_{\tau, 1} \boldsymbol{u}_{h}^{n}+\nabla p_{h}^{n}\right)\right) \\
\geq & \frac{C_{\mathrm{P}}^{2} \tau}{2 \nu} \sum_{n=2}^{N} \partial_{\tau, 1}\left\|\delta^{\frac{1}{2}}\left(\partial_{\tau, 1} \boldsymbol{u}_{h}^{n}+\nabla p_{h}^{n}\right)\right\|_{H}^{2}-\frac{C_{\mathrm{P}}^{2} \tau}{\nu} \sum_{n=2}^{N}\left(\partial_{\tau, 1} \boldsymbol{f}^{n}, \delta\left(\partial_{\tau, 1} \boldsymbol{u}_{h}^{n}+\nabla p_{h}^{n}\right)\right) \\
\geq & -\frac{C_{\mathrm{P}}^{2}}{2 \nu}\left\|\delta^{\frac{1}{2}}\left(\partial_{\tau, 1} \boldsymbol{u}^{1}+\nabla p_{h}^{1}\right)\right\|_{H}^{2}-\frac{C_{\mathrm{P}}^{4} \tau}{2 \nu^{2}} \sum_{n=2}^{N}\left\|\delta^{\frac{1}{2}} \partial_{\tau, 1} \boldsymbol{f}^{n}\right\|_{H}^{2} \\
& -\frac{\tau}{2} \sum_{n=2}^{N}\left\|\delta^{\frac{1}{2}}\left(\nabla p_{h}^{n}+\partial_{\tau, 1} \boldsymbol{u}_{h}^{n}\right)\right\|_{H}^{2} \cdot
\end{aligned}
$$

For $I_{4}$ we note that, from (5) with $q_{h}=0$, we have $(n \geq 2)$

$$
-\left(\partial_{\tau, 1}\left(\partial_{\tau, 1} \boldsymbol{u}_{h}^{n}+\nabla p_{h}^{n}-\boldsymbol{f}^{n}\right), \boldsymbol{v}_{h}\right)=\nu\left(\nabla \partial_{\tau, 1} \boldsymbol{u}_{h}^{n}, \nabla \boldsymbol{v}_{h}\right) .
$$

Hence, testing this expression with $\boldsymbol{v}_{h}=C_{\mathrm{P}}^{2} \nu^{-1} \tau \delta \partial_{\tau, 1} \boldsymbol{u}_{h}^{n}$ and summing over $n=2, \ldots, N$, yields

$$
I_{4}=\frac{C_{\mathrm{P}}^{2} \tau}{\nu} \sum_{n=2}^{N}\left\|\delta^{\frac{1}{2}} \partial_{\tau, 1} \boldsymbol{u}_{h}^{n}\right\|_{V}^{2} \geq 0 .
$$

Therefore, inserting the estimations of (26) and (27) into (25), gives

$$
\begin{aligned}
I_{1} \geq & -\frac{C_{\mathrm{P}}^{2}}{2 \nu}\left\|\delta^{\frac{1}{2}}\left(\partial_{\tau, 1} \boldsymbol{u}_{h}^{1}+\nabla p_{h}^{1}\right)\right\|_{H}^{2}-\frac{C_{\mathrm{P}}^{4} \tau}{2 \nu^{2}} \sum_{n=2}^{N}\left\|\delta^{\frac{1}{2}} \partial_{\tau, 1} \boldsymbol{f}^{n}\right\|_{H}^{2} \\
& -\frac{\tau}{2} \sum_{n=2}^{N}\left\|\delta^{\frac{1}{2}}\left(\nabla p_{h}^{n}+\partial_{\tau, 1} \boldsymbol{u}_{h}^{n}\right)\right\|_{H}^{2} .
\end{aligned}
$$

It now remains to derive a bound for $I_{2}$, which corresponds to the first time-step. We have

$$
I_{2}=-\underbrace{\frac{C_{\mathrm{P}}^{2}}{\nu}\left(p_{h}^{1}, \nabla \cdot \boldsymbol{u}_{h}^{1}\right)}_{I_{5}}+\underbrace{\frac{C_{\mathrm{P}}^{2}}{\nu}\left(p_{h}^{1}, \nabla \cdot \boldsymbol{u}_{h}^{0}\right)}_{I_{6}} .
$$

Term $I_{5}$ can be controlled using an argument similar to the one used to estimate $I_{1}$. We first note that, from (5) with $\boldsymbol{v}_{h}=0$, for $n=1$ we have

$$
-\left(q_{h}, \nabla \cdot \boldsymbol{u}_{h}^{1}\right)=\left(\partial_{\tau, 1} \boldsymbol{u}_{h}^{1}+\nabla p_{h}^{1}-\boldsymbol{f}^{1}, \delta \nabla q_{h}\right) .
$$

Hence, taking $q_{h}=C_{\mathrm{P}}^{2} \nu^{-1} p_{h}^{1}$, we get

$$
\begin{aligned}
I_{5}= & \underbrace{\frac{C_{\mathrm{P}}^{2}}{\nu}\left(\partial_{\tau, 1} \boldsymbol{u}_{h}^{1}+\nabla p_{h}^{1}-\boldsymbol{f}^{1}, \delta\left(\partial_{\tau, 1} \boldsymbol{u}_{h}^{1}+\nabla p_{h}^{1}\right)\right)}_{I_{7}} \\
& -\underbrace{\frac{C_{\mathrm{P}}^{2}}{\nu}\left(\partial_{\tau, 1} \boldsymbol{u}_{h}^{1}+\nabla p_{h}^{1}-\boldsymbol{f}^{1}, \delta \partial_{\tau, 1} \boldsymbol{u}_{h}^{1}\right)}_{I_{8}} .
\end{aligned}
$$


Term $I_{7}$ is estimated as follows

$$
\begin{aligned}
I_{7} & =\frac{C_{\mathrm{P}}^{2}}{\nu}\left(\partial_{\tau, 1} \boldsymbol{u}_{h}^{1}+\nabla p_{h}^{1}, \delta\left(\partial_{\tau, 1} \boldsymbol{u}_{h}^{1}+\nabla p_{h}^{1}\right)\right)-\frac{C_{\mathrm{P}}^{2}}{\nu}\left(\boldsymbol{f}^{1}, \delta\left(\partial_{\tau, 1} \boldsymbol{u}_{h}^{1}+\nabla p_{h}^{1}\right)\right) \\
& \geq \frac{3 C_{\mathrm{P}}^{2}}{4 \nu}\left\|\delta^{\frac{1}{2}}\left(\partial_{\tau, 1} \boldsymbol{u}_{h}^{1}+\nabla p_{h}^{1}\right)\right\|_{H}^{2}-\frac{C_{\mathrm{P}}^{2}}{\nu}\left\|\delta^{\frac{1}{2}} \boldsymbol{f}^{1}\right\|_{H}^{2} .
\end{aligned}
$$

For $I_{8}$, we take $n=1, q_{h}=0$ and $\boldsymbol{v}_{h}=C_{\mathrm{P}}^{2} \nu^{-1} \delta \partial_{\tau, 1} \boldsymbol{u}_{h}^{1}$ in (5), which yields

$$
\begin{aligned}
I_{8}= & \frac{C_{\mathrm{P}}^{2} \delta}{\nu}\left(\nu \boldsymbol{\nabla} \boldsymbol{u}_{h}^{1}, \boldsymbol{\nabla} \partial_{\tau, 1} \boldsymbol{u}_{h}^{1}\right)=\frac{C_{\mathrm{P}}^{2} \delta}{2} \partial_{\tau, 1}\left\|\nabla \boldsymbol{u}_{h}^{1}\right\|_{H}^{2}+\frac{C_{\mathrm{P}}^{2} \tau}{2}\left\|\delta^{\frac{1}{2}} \partial_{\tau, 1} \boldsymbol{\nabla} \boldsymbol{u}_{h}^{1}\right\|_{H}^{2} \\
& \geq-\frac{C_{\mathrm{P}}^{2} \delta}{2 \tau}\left\|\boldsymbol{\nabla} \boldsymbol{u}_{h}^{0}\right\|_{H}^{2} .
\end{aligned}
$$

As a result,

$$
I_{5} \geq \frac{3 C_{\mathrm{P}}^{2}}{4 \nu}\left\|\delta^{\frac{1}{2}}\left(\partial_{\tau, 1} \boldsymbol{u}_{h}^{1}+\nabla p_{h}^{1}\right)\right\|_{H}^{2}-\frac{C_{\mathrm{P}}^{2}}{\nu}\left\|\delta^{\frac{1}{2}} \boldsymbol{f}^{1}\right\|_{H}^{2}-\frac{C_{\mathrm{P}}^{2} \delta}{2 \tau}\left\|\nabla \boldsymbol{u}_{h}^{0}\right\|_{H}^{2} .
$$

The estimation of $I_{6}$ depends on the choice of the discrete initial velocity $\boldsymbol{u}_{h}^{0}$. Let us first consider the case $\boldsymbol{u}_{h}^{0}=\mathcal{I}_{h} \boldsymbol{u}_{0}$. Since $\boldsymbol{\nabla} \cdot \boldsymbol{u}_{0}=0$, we have

$$
I_{6}=\frac{C_{\mathrm{P}}^{2}}{\nu}\left(p_{h}^{1}, \boldsymbol{\nabla} \cdot\left(\mathcal{I}_{h}^{0} \boldsymbol{u}_{0}-\boldsymbol{u}_{0}\right)\right) \leq \frac{C_{\mathcal{I}}^{2}}{2 \nu} h^{2}\left\|p_{h}^{1}\right\|_{Q}^{2}+\frac{C_{\mathrm{P}}^{4}}{2}\left|\boldsymbol{u}_{0}\right|_{2, \Omega}^{2}
$$

Therefore, inserting the estimations 30 and 31 into 29 , gives

$$
\begin{aligned}
I_{2} \geq & \frac{3 C_{\mathrm{P}}^{2}}{4 \nu}\left\|\delta^{\frac{1}{2}}\left(\partial_{\tau, 1} \boldsymbol{u}_{h}^{1}+\nabla p_{h}^{1}\right)\right\|_{H}^{2}-\frac{C_{\mathrm{P}}^{2}}{\nu}\left\|\delta^{\frac{1}{2}} \boldsymbol{f}^{1}\right\|_{H}^{2}-\frac{C_{\mathrm{P}}^{4}}{2}\left|\boldsymbol{u}_{0}\right|_{2, \Omega}^{2} \\
& -\frac{C_{\mathrm{P}}^{2} \delta}{2 \tau}\left\|\nabla \boldsymbol{u}_{h}^{0}\right\|_{H}^{2}-\frac{C_{\mathcal{I}}^{2} \delta}{2 \gamma}\left\|p_{h}^{1}\right\|_{Q}^{2} .
\end{aligned}
$$

The stability estimate 20 then follows by applying 23 to 22 and inserting (28) and (32) into (24).

We will now choose the initial data as the reduced Ritz-projection (8) and show that this choice allows for less regular initial data. If $\boldsymbol{u}_{h}^{0}=\widetilde{\boldsymbol{R}}_{h} \boldsymbol{u}_{0}$, we use (8), with $\boldsymbol{v}_{h}=\mathbf{0}$ and $q_{h}=C_{\mathrm{P}}^{2} \nu^{-1} p_{h}^{1}$, to estimate term $I_{6}$ as follows

$$
\begin{aligned}
I_{6}= & \frac{C_{\mathrm{P}}^{2}}{\nu}\left(p_{h}^{1}, \boldsymbol{\nabla} \cdot \widetilde{\boldsymbol{R}}_{h} \boldsymbol{u}_{0}\right)=\frac{C_{\mathrm{P}}^{2} \delta}{\nu}\left(\boldsymbol{\nabla} \widetilde{P}_{h} \boldsymbol{u}_{0}, \boldsymbol{\nabla} p_{h}^{1}\right) \\
= & \frac{C_{\mathrm{P}}^{2} \delta}{\nu}\left(\boldsymbol{\nabla} \widetilde{P}_{h} \boldsymbol{u}_{0}, \boldsymbol{\nabla} p_{h}^{1}+\partial_{\tau, 1} \boldsymbol{u}_{h}^{1}\right)-\frac{C_{\mathrm{P}}^{2} \delta}{\nu}\left(\boldsymbol{\nabla} \widetilde{P}_{h} \boldsymbol{u}_{0}, \partial_{\tau, 1} \boldsymbol{u}_{h}^{1}\right) \\
\geq & -\frac{C_{\mathrm{P}}^{2}}{\nu}\left(1+\frac{\delta}{2 \tau}\right)\left\|\delta^{\frac{1}{2}} \boldsymbol{\nabla} \widetilde{P}_{h} \boldsymbol{u}_{0}\right\|_{H}^{2}-\frac{C_{\mathrm{P}}^{2}}{4 \nu}\left\|\delta^{\frac{1}{2}}\left(\boldsymbol{\nabla} p_{h}^{1}+\partial_{\tau, 1} \boldsymbol{u}^{1}\right)\right\|_{H}^{2} \\
& -\frac{C_{\mathrm{P}}^{2} \tau}{2 \nu}\left\|\partial_{\tau, 1} \boldsymbol{u}_{h}^{1}\right\|_{H}^{2},
\end{aligned}
$$

and hence, 32 becomes

$$
\begin{array}{r}
I_{2} \geq \frac{C_{\mathrm{P}}^{2}}{2 \nu}\left\|\delta^{\frac{1}{2}}\left(\partial_{\tau, 1} \boldsymbol{u}_{h}^{1}+\nabla p_{h}^{1}\right)\right\|_{H}^{2}-\frac{C_{\mathrm{P}}^{2}}{\nu}\left\|\delta^{\frac{1}{2}} \boldsymbol{f}^{1}\right\|_{H}^{2}-\frac{C_{\mathrm{P}}^{2} \delta}{2 \tau}\left\|\nabla \boldsymbol{u}_{h}^{0}\right\|_{H}^{2} \\
-\frac{C_{\mathrm{P}}^{2}}{\nu}\left(1+\frac{\delta}{2 \tau}\right)\left\|\delta^{\frac{1}{2}} \nabla \widetilde{P}_{h} \boldsymbol{u}_{0}\right\|_{H}^{2}-\frac{C_{\mathrm{P}}^{2} \tau}{2 \nu}\left\|\partial_{\tau, 1} \boldsymbol{u}_{h}^{1}\right\|_{H}^{2} .
\end{array}
$$


The stability estimate (21) then follows by applying $(23)$ to $(22)$ and inserting (28) and (33) into (24), after having applied the stability estimate for the Ritzprojection $(9) . \diamond$

Remark 4.6 Note that, contrary to the case of symmetric stabilization methods [9], the specific Ritz-projection (8) does not remove the small time-step pressure stability. It only enables the choice of less regular initial data.

\section{Convergence analysis}

The next theorem provides an optimal a priori error estimate for the velocities for the case of the backward differentiation formulas.

Theorem 5.1 Let $\left\{\left(\boldsymbol{u}_{h}^{n}, p_{h}^{n}\right)\right\}_{n=\kappa}^{N}$ be given by (5). Then, under the stability conditions given in Lemma 4.1. we have

$$
\begin{aligned}
&\left\|\boldsymbol{u}_{h}^{N}-\boldsymbol{u}\left(t^{N}\right)\right\|_{H}^{2}+\tau \sum_{n=\kappa}^{N}\left\|\boldsymbol{u}_{h}^{n}-\boldsymbol{u}\left(t^{n}\right)\right\|_{V}^{2} \\
&+\tau \sum_{n=\kappa}^{N}\left(\delta\left\|\partial_{\tau, \kappa}\left(\boldsymbol{u}_{h}^{n}-\boldsymbol{u}\left(t^{n}\right)\right)+\nabla\left(p_{h}^{n}-p\left(t^{n}\right)\right)\right\|_{H}^{2}\right) \\
& \leq C_{\nu, \boldsymbol{u}}\left(h^{2 k}+\tau^{2 \kappa}\right)+\sum_{j=0}^{\kappa-1}\left\|\boldsymbol{u}_{h}^{j}-\boldsymbol{u}\left(t^{j}\right)\right\|_{H}^{2}
\end{aligned}
$$

and

$$
\sup _{\kappa \leq n \leq N}\left\|\boldsymbol{u}_{h}^{n}-\boldsymbol{u}\left(t^{n}\right)\right\|_{H}^{2} \lesssim C_{\nu, \boldsymbol{u}}\left(h^{2(k+1)}+\tau^{2 \kappa}\right)+\sum_{j=0}^{\kappa-1}\left\|\boldsymbol{u}_{h}^{j}-\boldsymbol{u}\left(t^{j}\right)\right\|_{H}^{2},
$$

where $C_{\nu, \boldsymbol{u}}>0$ depends on $\nu$ and on Sobolev norms of $\boldsymbol{u}$.

Proof. Decompose the error as

$$
\boldsymbol{u}\left(t^{n}\right)-\boldsymbol{u}_{h}^{n}=\underbrace{\boldsymbol{u}\left(t^{n}\right)-\boldsymbol{R}_{h}\left(\boldsymbol{u}\left(t^{n}\right), p\left(t^{n}\right)\right)}_{\boldsymbol{\eta}^{n}}+\underbrace{\boldsymbol{R}_{h}\left(\boldsymbol{u}\left(t^{n}\right), p\left(t^{n}\right)\right)-\boldsymbol{u}_{h}^{n}}_{\boldsymbol{\theta}_{h}^{n}}=\boldsymbol{\eta}^{n}+\boldsymbol{\theta}_{h}^{n}
$$

and

$$
p\left(t^{n}\right)-p_{h}^{n}=\underbrace{p\left(t^{n}\right)-P_{h}\left(\boldsymbol{u}\left(t^{n}\right), p\left(t^{n}\right)\right)}_{\zeta^{n}}+\underbrace{P_{h}\left(\boldsymbol{u}\left(t^{n}\right), p\left(t^{n}\right)\right)-p_{h}}_{\xi_{h}^{n}}=\zeta^{n}-\xi_{h}^{n} .
$$

Consider the discrete error injected in the formulation (5), using Galerkin orthogonality in the first equality and the properties of the Ritz-projection in the second, we have

$$
\begin{aligned}
&\left(\partial_{\tau, \kappa} \boldsymbol{\theta}_{h}^{n}+\right.\left.\boldsymbol{\nabla} \xi_{h}^{n}, \boldsymbol{w}_{h}\right)-\left(\nu \boldsymbol{\Delta} \boldsymbol{\theta}_{h}^{n}, \delta \boldsymbol{\nabla} q_{h}\right)_{h}+a\left(\boldsymbol{\theta}_{h}^{n}, \boldsymbol{v}_{h}\right)+b\left(q_{h}, \boldsymbol{\theta}_{h}^{n}\right) \\
&=-\left(\boldsymbol{\nabla} \zeta^{n}, \boldsymbol{w}_{h}\right)+\left(\nu \boldsymbol{\Delta} \boldsymbol{\eta}^{n}, \delta \boldsymbol{\nabla} q_{h}\right)_{h}-a\left(\boldsymbol{\eta}^{n}, \boldsymbol{v}_{h}\right)-b\left(q_{h}, \boldsymbol{\eta}^{n}\right) \\
& \quad+\left(\partial_{\tau, \kappa} \boldsymbol{R}_{h}\left(\boldsymbol{u}\left(t^{n}\right), p\left(t^{n}\right)\right)-\partial_{t} \boldsymbol{u}\left(t^{n}\right), \boldsymbol{w}_{h}\right) \\
&=\left(\partial_{\tau, \kappa} \boldsymbol{R}_{h}\left(\boldsymbol{u}\left(t^{n}\right), p\left(t^{n}\right)\right)-\partial_{\tau, \kappa} \boldsymbol{u}\left(t^{n}\right)+\partial_{\tau, \kappa} \boldsymbol{u}\left(t^{n}\right)-\partial_{t} \boldsymbol{u}\left(t^{n}\right), \boldsymbol{w}_{h}\right) .
\end{aligned}
$$


It follows that the functions $\left\{\boldsymbol{\theta}_{h}^{n}, \xi_{h}^{n}\right\}_{n=1}^{N}$ satisfies the formulation (5) with $\boldsymbol{\theta}_{h}^{0}=$ $\boldsymbol{R}_{h}\left(\boldsymbol{u}_{0}, p(0)\right)-\boldsymbol{u}_{h}^{0}$ and source term

$$
\boldsymbol{f}^{n} \stackrel{\text { def }}{=} \partial_{\tau, \kappa} \boldsymbol{R}_{h}\left(\boldsymbol{u}\left(t^{n}\right), p\left(t^{n}\right)\right)-\partial_{\tau, \kappa} \boldsymbol{u}\left(t^{n}\right)+\partial_{\tau, \kappa} \boldsymbol{u}\left(t^{n}\right)-\partial_{t} \boldsymbol{u}\left(t^{n}\right) .
$$

Applying now the stability estimate of Lemma 4.1 to the perturbation equation (34) we may write

$$
\begin{aligned}
& \left\|\boldsymbol{\theta}_{h}^{N}\right\|_{H}^{2}+\tau \sum_{n=\kappa}^{N}\left(\left\|\delta^{\frac{1}{2}}\left(\partial_{\tau, \kappa} \boldsymbol{\theta}_{h}^{n}+\nabla \xi_{h}^{n}\right)\right\|_{H}^{2}+\left\|\boldsymbol{\theta}_{h}^{n}\right\|_{V}^{2}\right) \\
\lesssim & \left(\delta+\frac{C_{\mathrm{P}}^{2}}{\nu}\right) \tau \sum_{n=\kappa}^{N}\left\|\partial_{\tau, \kappa} \boldsymbol{\eta}^{n}+\partial_{\tau, \kappa} \boldsymbol{u}\left(t^{n}\right)-\partial_{t} \boldsymbol{u}\left(t^{n}\right)\right\|_{H}^{2}+\sum_{n=0}^{\kappa-1}\left(\left\|\boldsymbol{\theta}_{h}^{n}\right\|_{H}^{2}+\delta\left\|\boldsymbol{\theta}_{h}^{n}\right\|_{V}^{2}\right) .
\end{aligned}
$$

We conclude by applying the estimates for the Ritz projection and standard truncation error estimates yielding

$$
\begin{aligned}
\tau \sum_{n=\kappa}^{N} \| \partial_{\tau, \kappa} \boldsymbol{\eta}^{n}+\partial_{\tau, \kappa} & \boldsymbol{u}\left(t^{n}\right)-\partial_{t} \boldsymbol{u}\left(t^{n}\right) \|_{H}^{2} \\
& \lesssim h^{2(k+1)} \int_{0}^{T}\left|\partial_{t} \boldsymbol{u}(t)\right|_{k+1}^{2} \mathrm{~d} t+\tau^{2 \kappa} \int_{0}^{T}\left\|\partial_{t}^{\kappa+1} \boldsymbol{u}(t)\right\|_{H}^{2} \mathrm{~d} t,
\end{aligned}
$$

which completes the proof. $\diamond$

An optimal a priori error estimate for the pressure follows in a similar fashion, by combining the above result with the pressure stability estimate provided by Theorem 4.5 applied to the perturbation equation (34). The result is stated in the next corollary.

Corollary 5.2 Let $\left\{\left(\boldsymbol{u}_{h}^{n}, p_{h}^{n}\right)\right\}_{n=1}^{N}$ be given by (5) with $\boldsymbol{u}_{h}^{0}=\widetilde{\boldsymbol{R}}_{h}\left(\boldsymbol{u}_{0}\right), \kappa=1$ and $k=1$ in (4). Assume that $\delta \lesssim \tau$. Then, there holds

$$
\tau \sum_{n=1}^{N}\left\|p_{h}^{n}-p\left(t^{n}\right)\right\|_{Q}^{2} \leq C_{\nu, \boldsymbol{u}, p}\left(h^{2}+\tau^{2}\right)
$$

where $C_{\nu, \boldsymbol{u}, p}$ depends on $\nu$ and on Sobolev norms of $\boldsymbol{u}$ and $p$.

\section{A weakly consistent PSPG-method using a re- constructed Laplacian}

As we have seen above (see Remark 4.2 ) it is the nonconformity of the approximation of the Laplacian operator that gives rise to the velocity instabilities. We will now recall a method proposed by Bochev and Gunzburger in 4, Section 5] which was shown to have improved properties compared to the standard PSPG method. The idea is to use a variational projection to reconstruct the Laplacian operator. Here we propose a slight modification in which the boundary conditions as well are built into the reconstruction using a Nitsche type argument. 
For all $\boldsymbol{v} \in\left[H^{\frac{3}{2}+\epsilon}(\Omega)\right]^{d} \cup W_{h}$ (with $\epsilon>0$ ), the discrete Laplacian of $\boldsymbol{v}$ is defined as $\boldsymbol{\Delta}_{h} \boldsymbol{v} \in W_{h}$ such that

$$
-\left(\boldsymbol{\Delta}_{h} \boldsymbol{v}, \boldsymbol{w}_{h}\right)=\left(\boldsymbol{\nabla} \boldsymbol{v}, \boldsymbol{\nabla} \boldsymbol{w}_{h}\right)+\left(\boldsymbol{\xi}(\boldsymbol{v}), \boldsymbol{w}_{h}\right)_{\partial \Omega}+\left(\boldsymbol{\xi}\left(\boldsymbol{w}_{h}\right), \boldsymbol{v}\right)_{\partial \Omega}
$$

for all $\boldsymbol{w}_{h} \in W_{h}$, where

$$
\boldsymbol{\xi}(\boldsymbol{v}) \stackrel{\text { def }}{=}-\boldsymbol{n} \cdot \boldsymbol{\nabla} \boldsymbol{v}+\frac{\gamma_{\mathrm{bc}}}{2 h} \boldsymbol{v}
$$

with $\gamma_{\mathrm{bc}}>0$ a given (large enough) dimensionless parameter.

The formulation then reads: For $n \geq \kappa$, find $\left(\boldsymbol{u}_{h}^{n}, p_{h}^{n}\right) \in W_{h} \times Q_{h}$ such that

$$
\left\{\begin{array}{l}
\left(\partial_{\tau, \kappa} \boldsymbol{u}_{h}^{n}+\nabla p_{h}^{n}-\nu \boldsymbol{\Delta}_{h} \boldsymbol{u}_{h}^{n}, \boldsymbol{v}_{h}\right)=\left(\boldsymbol{f}^{n}, \boldsymbol{v}_{h}\right), \\
\left(\nabla \cdot \boldsymbol{u}_{h}^{n}, q_{h}\right)-\left(\boldsymbol{u}_{h}^{n} \cdot \boldsymbol{n}, q_{h}\right)_{\partial \Omega}+\left(\partial_{\tau, \kappa} \boldsymbol{u}_{h}^{n}+\nabla p_{h}^{n}-\nu \boldsymbol{\Delta}_{h} \boldsymbol{u}_{h}^{n}-\pi_{h} \boldsymbol{f}^{n}, \delta \boldsymbol{\nabla} q_{h}\right)=0, \\
\left(-\boldsymbol{\Delta}_{h} \boldsymbol{u}_{h}^{n}, \boldsymbol{w}_{h}\right)=\left(\boldsymbol{\nabla} \boldsymbol{u}_{h}^{n}, \boldsymbol{\nabla} \boldsymbol{w}_{h}\right)-\left(\boldsymbol{\xi}\left(\boldsymbol{u}_{h}^{n}\right), \boldsymbol{w}_{h}\right)_{\partial \Omega}-\left(\boldsymbol{\xi}\left(\boldsymbol{w}_{h}\right), \boldsymbol{u}_{h}^{n}\right)_{\partial \Omega}
\end{array}\right.
$$

for all $\left(\boldsymbol{v}_{h}, q_{h}, \boldsymbol{w}_{h}\right) \in W_{h} \times Q_{h} \times W_{h}$.

Note that here the boundary conditions are not built into the approximation space and, by the definition of the reconstruction 35$]_{3}$, the first line of equation (35) is equivalent to the standard weak formulation with Nitsche type boundary conditions (see, e.g., [10]). The reconstruction changes only the formulation of the continuity equation. Indeed observe that, compared to the standard PSPG method (5), $\boldsymbol{\Delta} \boldsymbol{u}_{h}$ has been replaced by $\boldsymbol{\Delta}_{h} \boldsymbol{u}_{h}$ and $\boldsymbol{f}^{n}$ by its $L^{2}$-projection $\pi_{h} \boldsymbol{f}^{n}$ leading to a formulation that is weakly consistent instead of strongly.

For this formulation it is straightforward to show that the stability estimate of Lemma 4.1 holds, without any constraint on the discretization parameters and regardless of the polynomial order. Indeed, the main idea consists of first noting that, the critical term $(11)$ becomes

$$
I_{1}=-\delta\left(\nu \boldsymbol{\Delta}_{h} \boldsymbol{u}_{h}^{n}, \partial_{\tau, \kappa} \boldsymbol{u}_{h}^{n}+\nabla p_{h}^{n}\right)_{h} .
$$

On the other hand, by using standard inverse and trace inequalities, we can get the following inverse estimate for the reconstructed Laplacian operator

$$
\left\|\nu^{\frac{1}{2}} \boldsymbol{\Delta}_{h} \boldsymbol{v}_{h}\right\|_{H} \lesssim \frac{1}{h}\left\|\boldsymbol{v}_{h}\right\|_{W_{h}}
$$

for all $\boldsymbol{v}_{h} \in W_{h}$, where

$$
\left\|\boldsymbol{v}_{h}\right\|_{W_{h}}^{2} \stackrel{\text { def }}{=}\left\|\boldsymbol{v}_{h}\right\|_{V}^{2}+\frac{\gamma_{\mathrm{bc}} \nu}{h}\left\|\boldsymbol{v}_{h}\right\|_{0, \partial \Omega}^{2}
$$

is the new velocity coercivity-norm associated to the formulation (35). The term $I_{1}$ can therefore be estimated as follows, using (37),

$$
\begin{aligned}
I_{1} & \leq \frac{\delta \nu}{2 \epsilon}\left\|\nu^{\frac{1}{2}} \boldsymbol{\Delta}_{h} \boldsymbol{u}_{h}^{n}\right\|_{H}^{2}+\frac{\epsilon}{2}\left\|\delta^{\frac{1}{2}}\left(\partial_{\tau, \kappa} \boldsymbol{u}_{h}^{n}+\nabla p_{h}^{n}\right)\right\|_{H}^{2} \\
& \lesssim \frac{\gamma}{2 \epsilon}\left\|\boldsymbol{u}_{h}^{n}\right\|_{W_{h}}^{2}+\frac{\epsilon}{2}\left\|\delta^{\frac{1}{2}}\left(\partial_{\tau, \kappa} \boldsymbol{u}_{h}^{n}+\nabla p_{h}^{n}\right)\right\|_{H}^{2}
\end{aligned}
$$

with $\epsilon>0$, where the right-hand side is simply controlled by choosing $\gamma, \epsilon$ sufficiently small and by using the standard coercivity argument for Nitsche's method [17, 10]. 
Note that the pressure analysis may be performed as well, leading to unconditional stability, provided the initial condition is chosen as a suitably chosen Ritz-projection and under some assumptions on the regularity of $\boldsymbol{f}$. Instead of proceeding with this analysis, here we will chose a simpler way of showing the improved properties of the formulation 35 . Indeed we show that the formulation (35) is equivalent to the quasi static orthogonal subscale method proposed by Codina and Blasco [11, which was analysed in the transient case in [9].

\subsection{Relation to the Orthogonal Subscale Stabilization}

Taking $\boldsymbol{v}_{h}=\delta \pi_{h} \boldsymbol{\nabla} q_{h}$ in $35{ }_{1}$ yields

$$
\left(\partial_{\tau, \kappa} \boldsymbol{u}_{h}^{n}+\nabla p_{h}^{n}-\nu \boldsymbol{\Delta}_{h} \boldsymbol{u}_{h}^{n}, \delta \pi_{h} \boldsymbol{\nabla} q_{h}\right)=\left(\pi_{h} \boldsymbol{f}^{n}, \delta \pi_{h} \boldsymbol{\nabla} q_{h}\right)
$$

By subtracting this equality from $\sqrt{35)_{2}}$ we then obtain

$$
\begin{array}{r}
\left(\boldsymbol{\nabla} \cdot \boldsymbol{u}_{h}^{n}, q_{h}\right)-\left(\boldsymbol{u}_{h}^{n} \cdot \boldsymbol{n}, q_{h}\right)_{\partial \Omega}+\left(\partial_{\tau, \kappa} \boldsymbol{u}_{h}^{n}+\nabla p_{h}^{n}-\nu \boldsymbol{\Delta}_{h} \boldsymbol{u}_{h}^{n}, \delta\left(\nabla q_{h}-\pi_{h} \boldsymbol{\nabla} q_{h}\right)\right) \\
=\delta\left(\pi_{h} \boldsymbol{f}^{n}, \boldsymbol{\nabla} q_{h}-\pi_{h} \boldsymbol{\nabla} q_{h}\right) .
\end{array}
$$

At last, from the orthogonality of the $L^{2}$-projection $\pi_{h}$, we have

$$
\left(\boldsymbol{\nabla} \cdot \boldsymbol{u}_{h}^{n}, q_{h}\right)-\left(\boldsymbol{u}_{h}^{n} \cdot \boldsymbol{n}, q_{h}\right)_{\partial \Omega}+\delta\left(\left(I-\pi_{h}\right) \boldsymbol{\nabla} p_{h}^{n},\left(I-\pi_{h}\right) \boldsymbol{\nabla} q_{h}\right)=0
$$

for all $q_{h} \in Q_{h}$. This is exactly the incompressibility equation of the (quasistatic) Orthogonal Subscale Stabilization (OSS), see [11, 1]. We conclude that the OSS method and the modified PSPG method of [4, Section 5] are equivalent methods up to the boundary conditions. If Nitsche type boundary conditions are used in the former case and integrated in the reconstruction in the latter, the two formulations coincide. By this equivalence a full analysis of the method (35) with first and second order backward differentiation (or Crank-Nicolson time-discretization) follows from the analysis of OSS in [9].

\section{Numerical example}

The pressure instability of the PSPG method has been thoroughly investigated in 6]. In [9], we showed both stable and unstable solutions for symmetric stabilizations, depending on how the initial data was chosen. The velocity instability indicated by the theory above, however, has not been reported in the literature (to the best of our knowledge). This is probably because it is triggered by a coupling between the jump of the gradient and the time-derivative. Hence, in many cases the method remains stable since the gradient jumps are so small for high-order elements that the term remains bounded by the viscous dissipation and the dissipation of the time-discretization scheme. Here we give an example of the instability, when using the Crank-Nicolson scheme in time and third order polynomial approximations for both velocities and pressure in space. The computations were performed using the FreeFem ++ package [15. The computational domain was the unit square, with ten elements on each side. A Delaunay mesh was used. Following [9], the source term and boundary data 


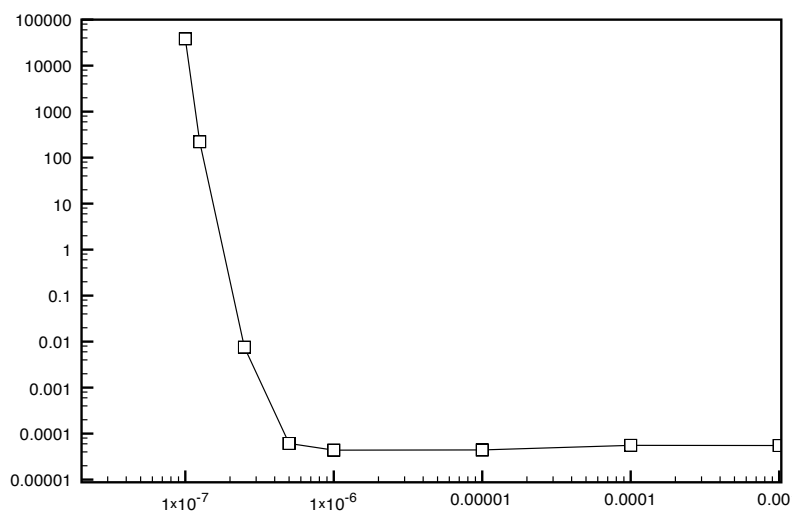

Figure 1: $l^{\infty}\left((0,200 \tau) ; L^{2}(\Omega)\right)$-norm error of the velocities versus time-step size $\tau$.

were chosen so that

$$
\begin{aligned}
& \boldsymbol{u}(x, y, t)=\left[\begin{array}{c}
\cos (t) \sin (\pi x-0.7) \sin (\pi y+0.2) \\
\cos (t) \cos (\pi x-0.7) \sin (\pi y+0.2)
\end{array}\right], \\
& p(x, y, t)=\cos (t)(\sin (x) \cos (y)+(\cos (1)-1) \sin (1)) .
\end{aligned}
$$

Starting from the timestep $\tau=10^{-3}$ we ran a sequence of simulations with decreasing time-step. For each timestep-size we took 200 steps, so that the final
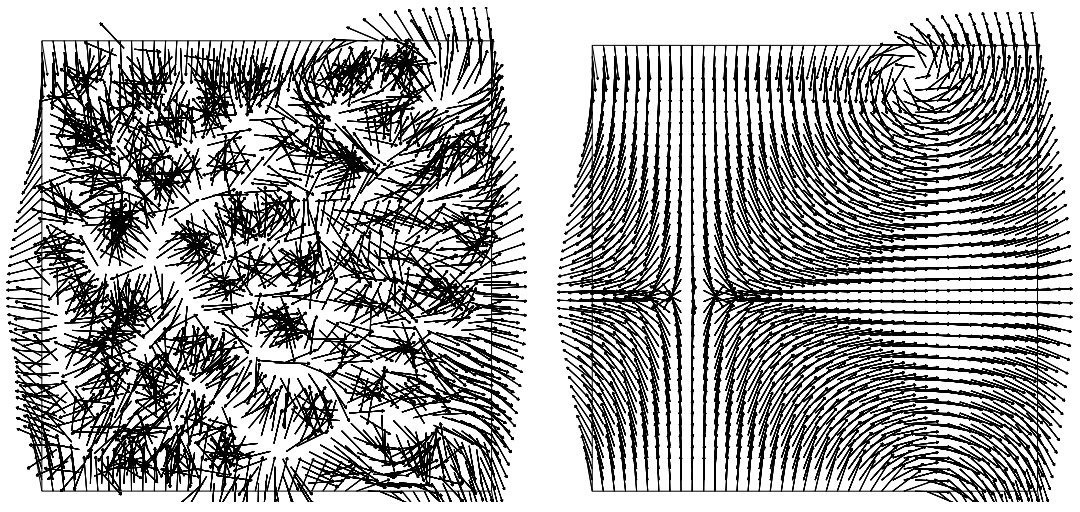

Figure 2: Velocity vector field after 50 timesteps, $\tau=2 \times 10^{-8}$. Left: the standard PSPG-method (6). Right: the method using reconstruction (35) and the Crank-Nicolson method for the time discretization.

time actually decreased for smaller steps. The error in the $l^{\infty}\left((0,200 \tau) ; L^{2}(\Omega)\right)$ norm against timestep-size is reported in Figure 1 . Note that for step-sizes down to $\tau=10^{-6}$ the method remains stable on this (shrinking) time interval. For $\tau<10^{-6}$ however a brutal loss of stability is observed. In the shown example $\gamma=0.075$, however we tried values of $\gamma$ down to $\gamma=5 \times 10^{-5}$ and the only effect was to postpone the onset of instability. Needless to say that, for such a small $\gamma$, the pressure was unstable. We have also verified numerically the stability of the method (35) (but using Crank-Nicolson for the discretization in time) on 
this example. We show the solutions of both cases (with and without Laplacian reconstruction) after 50 time-steps of size $2 \times 10^{-8}$ in Figure 2 The determining feature for stability in this case is the reconstruction. Indeed the solution remained stable also when the boundary conditions were imposed strongly.

\section{Acknowledgment}

This paper was written during research visits financed by the Alliance Programme and the British Council Proj. No. 09.007.

\section{References}

[1] S. Badia and R. Codina. On a multiscale approach to the transient Stokes problem: dynamic subscales and anisotropic space-time discretization. Appl. Math. Comput., 207(2):415-433, 2009.

[2] G. R. Barrenechea and J. Blasco. Pressure stabilization of finite element approximations of time-dependent incompressible flow problems. Comput. Methods Appl. Mech. Engrg., 197(1-4):219-231, 2007.

[3] Y. Bazilevs, L. Beirão da Veiga, J. A. Cottrell, T. J. R. Hughes, and G. Sangalli. Isogeometric analysis: approximation, stability and error estimates for $h$-refined meshes. Math. Models Methods Appl. Sci., 16(7):1031-1090, 2006.

[4] P. Bochev and M. Gunzburger. An absolutely stable pressure-Poisson stabilized finite element method for the Stokes equations. SIAM J. Numer. Anal., 42(3):1189-1207 (electronic), 2004.

[5] P. B. Bochev, M. D. Gunzburger, and R. B. Lehoucq. On stabilized finite element methods for transient problems with varying time scales. 1st LNCC Meeting on Computational Modeling, Petropolis, Brasil, 9-13 August 2004.

[6] P. B. Bochev, M. D. Gunzburger, and R. B. Lehoucq. On stabilized finite element methods for the Stokes problem in the small time step limit. Internat. J. Numer. Methods Fluids, 53(4):573-597, 2007.

[7] P. B. Bochev, M. D. Gunzburger, and J. N. Shadid. On inf-sup stabilized finite element methods for transient problems. Comput. Methods Appl. Mech. Engrg., 193(15-16):1471-1489, 2004.

[8] E. Burman. Consistent SUPG-method for transient transport problems: stability and convergence. Comput. Methods Appl. Mech. Engrg., 199(1720):1114-1123, 2010.

[9] E. Burman and M. A. Fernández. Galerkin finite element methods with symmetric pressure stabilization for the transient Stokes equations: stability and convergence analysis. SIAM J. Numer. Anal., 47(1):409-439, 2008/09. 
[10] E. Burman, M. A. Fernández, and P. Hansbo. Continuous interior penalty finite element method for Oseen's equations. SIAM J. Numer. Anal., 44(3):1248-1274 (electronic), 2006.

[11] R. Codina and J. Blasco. A finite element formulation for the Stokes problem allowing equal velocity-pressure interpolation. Comput. Methods Appl. Mech. Engrg., 143(3-4):373-391, 1997.

[12] M. Crouzeix and V. Thomée. The stability in $L_{p}$ and $W_{p}^{1}$ of the $L_{2}$ projection onto finite element function spaces. Math. Comp., 48(178):521$532,1987$.

[13] A. Ern and J.-L. Guermond. Theory and practice of finite elements, volume 159 of Applied Mathematical Sciences. Springer-Verlag, New York, 2004.

[14] V. Girault and P.-A. Raviart. Finite element methods for Navier-Stokes equations, volume 5 of Springer Series in Computational Mathematics. Springer-Verlag, Berlin, 1986.

[15] F. Hecht, O. Pironneau, A. Le Hyaric, and K. Ohtsuka. FreeFem $++v$. 2.11. User's Manual. LJLL, University of Paris 6.

[16] T. J. R. Hughes, L. P. Franca, and M. Balestra. A new finite element formulation for computational fluid dynamics. V. Circumventing the BabuškaBrezzi condition: a stable Petrov-Galerkin formulation of the Stokes problem accommodating equal-order interpolations. Comput. Methods Appl. Mech. Engrg., 59(1):85-99, 1986.

[17] J. Nitsche. Über ein Variationsprinzip zur Lösung von Dirichlet-Problemen bei Verwendung von Teilräumen, die keinen Randbedingungen unterworfen sind. Abh. Math. Sem. Univ. Hamburg, 36:9-15, 1971.

[18] M. Picasso and J. Rappaz. Stability of time-splitting schemes for the Stokes problem with stabilized finite elements. Numer. Methods Partial Differential Equations, 17(6):632-656, 2001.

[19] L. Tobiska and R. Verfürth. Analysis of a streamline diffusion finite element method for the Stokes and Navier-Stokes equations. SIAM J. Numer. Anal., 33(1):107-127, 1996. 
Unité de recherche INRIA Rocquencourt Domaine de Voluceau - Rocquencourt - BP 105 - 78153 Le Chesnay Cedex (France)

Unité de recherche INRIA Futurs : Parc Club Orsay Université - ZAC des Vignes 4, rue Jacques Monod - 91893 ORSAY Cedex (France)

Unité de recherche INRIA Lorraine : LORIA, Technopôle de Nancy-Brabois - Campus scientifique 615, rue du Jardin Botanique - BP 101 - 54602 Villers-lès-Nancy Cedex (France)

Unité de recherche INRIA Rennes : IRISA, Campus universitaire de Beaulieu - 35042 Rennes Cedex (France)

Unité de recherche INRIA Rhône-Alpes : 655, avenue de l'Europe - 38334 Montbonnot Saint-Ismier (France) Unité de recherche INRIA Sophia Antipolis : 2004, route des Lucioles - BP 93 - 06902 Sophia Antipolis Cedex (France) 